\title{
Nontransitive Decomposable Conjoint Measurement ${ }^{1}$
}

\author{
D. Bouyssou ${ }^{2}$ \\ M. Pirlot ${ }^{3}$ \\ CNRS - LAMSADE Faculté Polytechnique de Mons \\ Revised 16 January 2002 - Forthcoming in Journal of \\ Mathematical Psychology
}

\begin{abstract}
${ }^{1}$ We wish to thank M. Abdellaoui, J.-P. Doignon, Ch. Gonzales, Th. Marchant, P.P. Wakker and an anonymous referee for their very helpful suggestions and comments on earlier drafts of this text. The usual caveat applies. Denis Bouyssou gratefully acknowledges the support of the Centre de Recherche de l'ESSEC and the Brussels-Capital Region through a "Research in Brussels" action grant.

${ }^{2}$ LAMSADE, Université Paris Dauphine, Place du Maréchal de Lattre de Tassigny, F-75775 Paris Cedex 16, France, tel: +33 1440548 98, fax: +3314405 40 91, e-mail: bouyssou@lamsade.dauphine.fr.

${ }^{3}$ Faculté Polytechnique de Mons, 9, rue de Houdain, B-7000 Mons, Belgium, tel: +32 65 374682, fax: + 3265374689 , e-mail: pirlot@mathro.fpms.ac.be. Corresponding author.
\end{abstract}




\begin{abstract}
Traditional models of conjoint measurement look for an additive representation of transitive preferences. They have been generalized in two directions. Nontransitive additive conjoint measurement models allow for nontransitive preferences while retaining the additivity feature of traditional models. Decomposable conjoint measurement models are transitive but replace additivity by a mere decomposability requirement. This paper presents generalizations of conjoint measurement models combining these two aspects. This allows us to propose a simple axiomatic treatment that shows the pure consequences of several cancellation conditions used in traditional models. These nontransitve decomposable conjoint measurement models encompass a large number of aggregation rules that have been introduced in the literature.
\end{abstract}

Keywords: Conjoint measurement, Cancellations conditions, Nontransitive preferences, Decomposable models.

Suggested running title: Nontransitive Decomposable Conjoint Measurement 


\section{Introduction}

Given a binary relation $\succsim$ on a set $X=X_{1} \times X_{2} \times \cdots \times X_{n}$, the theory of conjoint measurement consists in finding conditions under which it is possible to build a homomorphism between this relational structure and a relational structure on $\mathbb{R}$. In traditional models of conjoint measurement $\succsim$ is supposed to be complete and transitive and the numerical representation is sought to be additive; the desired measurement model is such that:

$$
x \succsim y \Leftrightarrow \sum_{i=1}^{n} u_{i}\left(x_{i}\right) \geq \sum_{i=1}^{n} u_{i}\left(y_{i}\right)
$$

where $u_{i}$ are real-valued functions on the sets $X_{i}$ and it is understood that $x=\left(x_{1}, x_{2}, \ldots, x_{n}\right)$ and $y=\left(y_{1}, y_{2}, \ldots, y_{n}\right)$.

Many axiom systems have been proposed in order to obtain such a representation (Krantz, Luce, Suppes, \& Tversky, 1971; Wakker, 1989). Two main cases arise:

- When $X$ is finite (but of arbitrary cardinality), it is well-known that the system of axioms necessarily involves a denumerable number of cancellation conditions guaranteeing the existence of solutions to a system of (finitely many) linear inequalities through the use of various versions of the theorem of the alternative (see Scott (1964) or Krantz et al. (1971, Chapter 9). For recent contributions, see Fishburn $(1996,1997)$ ).

- When $X$ is infinite the picture changes provided that conditions are imposed in order to guarantee that the structure of $X$ is "close" to the structure of $\mathbb{R}$ and that $\succsim$ behaves consistently in this continuum; this is traditionally done using either an archimedean axiom together with some solvability assumption (Krantz et al., 1971, Chapter 6) or imposing some topological structure on $X$ and a continuity requirement on $\succsim$ (Debreu, 1960; Wakker, 1989). Under these conditions, it is wellknown that model (1) obtains using a finite - and limited - number of cancellation conditions (for recent contributions, see Gonzales (1996, 2000) and Karni and Safra (1998); for an alternative approach, extending the technique used in the finite case to the infinite one, see Jaffray (1974)). As opposed to the finite case, these structural assumptions allow us to obtain nice uniqueness results for model (1): the functions $u_{i}$ define interval scales with a common unit. 
In the finite case the axiom system is hardly interpretable and testable. In the infinite case, it is not always easy to separate the respective roles of the (unnecessary) structural assumptions from the (necessary) cancellation conditions as forcefully argued by Krantz et al. (1971, Chapter 9) and Furkhen and Richter (1991). One possible way out of this difficulty is to study more general models replacing additivity by a mere decomposability requirement. Krantz et al. (1971, Chapter 7) introduced the following decomposable model:

$$
x \succsim y \Leftrightarrow F\left(u_{1}\left(x_{1}\right), u_{2}\left(x_{2}\right), \ldots, u_{n}\left(x_{n}\right)\right) \geq F\left(u_{1}\left(y_{1}\right), u_{2}\left(y_{2}\right), \ldots, u_{n}\left(y_{n}\right)\right)
$$

where $F$ is increasing in all its arguments.

When $X$ is denumerable (i.e. finite or countably infinite), necessary and sufficient conditions for (2) consist in a transitivity and completeness requirement together with a single cancellation condition requiring that the preference between objects differing on a single attribute is independent from their common level on the remaining $n-1$ attributes. In the general case these conditions turn out to have identical implication when supplemented with the obviously necessary requirement that a numerical representation exists for $\succsim$. Though (2) may appear as exceedingly general when compared to (1), it allows us to deal with the finite and the infinite case in a unified way using a simple axiom system while imposing nontrivial restrictions on $\succsim$. The price to pay is that uniqueness results for model (2) are much less powerful than what they are for model (1), see Krantz et al. (1971, Chapter 7). It should be finally observed that proofs for model (2) are considerably simpler than they are for model (1). A wide variety of models "in between" (1) and (2) are studied in Luce, Krantz, Suppes, and Tversky (1990).

Both (1) and (2) imply that $\succsim$ is complete and transitive. Among many others, May (1954) and Tversky (1969) (see however the interpretation of Tversky's results in Iverson and Falmagne (1985)) have argued that the transitivity hypothesis is unlikely to hold when subjects are asked to compare objects evaluated on several attributes; more recently, Fishburn (1991a) has forcefully shown the need for models encompassing intransitivities (it should be noted that intransitive preferences have even attracted the attention of some economists, see e.g. Chipman (1971) or Kim and Richter (1986)). Tversky (1969) was one of the first to propose such a model generalizing (1), known as the additive difference model, in which:

$$
x \succsim y \Leftrightarrow \sum_{i=1}^{n} \Phi_{i}\left(u_{i}\left(x_{i}\right)-u_{i}\left(y_{i}\right)\right) \geq 0
$$


where $\Phi_{i}$ are increasing and odd functions.

It is clear that (3) allows for intransitive $\succsim$ but implies its completeness. When attention is restricted to the comparison of objects that only differ on one attribute, (3), as well as (2) and (1), implies that the preference between these objects is independent from their common level on the remaining $n-1$ attributes. This allows us to unambiguously define a preference relation on each attribute; it is clearly complete. Although model (3) can accommodate intransitive $\succsim$, a consequence of the increasingness of the $\Phi_{i}$ is that the preference relations defined on each attribute are transitive. This, in particular, excludes the possibility of any perception threshold on each attribute which would lead to an intransitive indifference relation on each attribute (on preference models with thresholds, allowing for intransitive indifference relations, we refer to Fishburn (1985), Pirlot and Vincke (1997), or Suppes, Krantz, Luce, and Tversky (1989)). Imposing that $\Phi_{i}$ are nondecreasing instead of being increasing allows for such a possibility. This gives rise to what Bouyssou (1986) called the "weak additive difference model".

As suggested by Bouyssou (1986), Fishburn (1990b, 1990a, 1991b) and Vind (1991), the subtractivity requirement in (3) can be relaxed. This leads to nontransitive additive conjoint measurement models in which:

$$
x \succsim y \Leftrightarrow \sum_{i=1}^{n} p_{i}\left(x_{i}, y_{i}\right) \geq 0
$$

where the $p_{i}$ are real-valued functions on $X_{i}^{2}$ and may have several additional properties (e.g. $p_{i}\left(x_{i}, x_{i}\right)=0$, for all $i \in\{1,2, \ldots, n\}$ and all $x_{i} \in X_{i}$ ).

This model is an obvious generalization of the (weak) additive difference model. It allows for intransitive and incomplete preference relations $\succsim$ as well as for intransitive and incomplete "partial preferences". An interesting specialization of (4) obtains when $p_{i}$ are required to be skew symmetric i.e. such that $p_{i}\left(x_{i}, y_{i}\right)=-p_{i}\left(y_{i}, x_{i}\right)$. This skew symmetric nontransitive additive conjoint measurement model implies the completeness of $\succsim$ and that the preference between objects only differing on some attributes is independent from their common level on the remaining attributes.

Fishburn (1991a) gives an excellent overview of these nontransitive models. Several axiom systems have been proposed to characterize them. Fishburn (1990b, 1991b) gives axioms for the skew symmetric version of (4) both in the finite and the infinite case. Necessary and sufficient conditions for a nonstandard version of (4) are presented in Fishburn (1992b). Vind (1991) 
gives axioms for (4) with $p_{i}\left(x_{i}, x_{i}\right)=0$ when $n \geq 4$. Bouyssou (1986) gives necessary and sufficient conditions for (4) with and without skew symmetry in the denumerable case when $n=2$.

The additive difference model (3) was axiomatized in Fishburn (1992a) in the infinite case when $n \geq 3$ and Bouyssou (1986) gives necessary and sufficient conditions for the weak additive difference model in the finite case when $n=2$. Related studies of nontransitive models include Croon (1984), Fishburn (1980), Luce (1978) and Nakamura (1997). The implications of these models for decision-making under uncertainty were explored in Fishburn (1990c) (for a different path to nontransitive models for decision making under risk and/or uncertainty see Fishburn $(1982,1988)$ ).

It should be noticed that even the weakest form of these models, i.e. (4) without skew symmetry, involves an addition operation. Therefore it is unsurprising that the difficulties that we mentioned concerning the axiomatic analysis of traditional models are still present here. Except in the special case in which $n=2$, this case relating more to ordinal than to conjoint measurement, the various axiom systems that have been proposed involve either:

- a denumerable set of cancellation conditions in the finite case or

- a finite number of cancellation conditions together with unnecessary structural assumptions in the general case (these structural assumptions generally allow us to obtain nice uniqueness results for (4): the functions $p_{i}$ are unique up to the multiplication by a common positive constant).

The nontransitive decomposable models that we study in this paper may be seen both as a generalization of (2) dropping transitivity and completeness and as a generalization of (4) dropping additivity. In their most general form they are of the type:

$$
x \succsim y \Leftrightarrow F\left(p_{1}\left(x_{1}, y_{1}\right), p_{2}\left(x_{2}, y_{2}\right), \ldots, p_{n}\left(x_{n}, y_{n}\right)\right) \geq 0
$$

where $F$ and $p_{i}$ may have several additional properties, e.g. $p_{i}\left(x_{i}, x_{i}\right)=0$ or $F$ nondecreasing or increasing in all its arguments.

This type of nontransitive decomposable conjoint models has been introduced by Goldstein (1991). They may be seen as exceedingly general. However we shall see that this model and its extensions: 
- imply substantive requirements on $\succsim$,

- may be axiomatized in a simple way avoiding the use of a denumerable number of conditions in the finite case and of unnecessary structural assumptions in the general case,

- permit to study the pure consequences of cancellation conditions in the absence of transitivity, completeness and structural requirements on $X$,

- are sufficiently general to include as particular cases many aggregation models that have been proposed in the literature.

Our project was to investigate how far it was possible to go in terms of numerical representations using a limited number of cancellation conditions without imposing any transitivity requirement on the preference relation and any structural assumptions on the set of objects. Rather surprisingly, as we shall see, such a poor framework allows us to go rather far.

It should be clear that, in models of type (5), numerical representations are quite unlikely to possess any "nice" uniqueness properties. This is all the more true that we will refrain in this paper from using unnecessary structural assumptions on the set of objects. Numerical representations are not studied here for their own sake; our results are not intended to provide clues on how to build them. We use them as a framework allowing us to understand the consequences of a number of requirements on $\succsim$.

The paper is organized as follows. We introduce our main definitions in section 2. We present the decomposable nontransitive conjoint measurement models to be studied in this paper and analyze some of their properties in section 3. We present our main results in section 4. A final section discusses our results and presents directions for future research.

\section{Definitions and Notation}

A binary relation $S$ on a set $A$ is a subset of $A \times A$; we write $a S b$ instead of $(a, b) \in S$. A binary relation $S$ on $A$ is said to be:

- reflexive if $[a S a]$,

- irreflexive if $[\operatorname{Not}(a S a)]$,

- complete if $[a S b$ or $b S a]$, 
- symmetric if $[a S b] \Rightarrow[b S a]$,

- asymmetric if $[a S b] \Rightarrow[\operatorname{Not}(b S a)]$,

- transitive if $[a S b$ and $b S c] \Rightarrow[a S c]$,

for all $a, b, c \in A$.

A weak order (resp. an equivalence) is a complete and transitive (resp. reflexive, symmetric and transitive) binary relation. If $S$ is an equivalence on $A, A / S$ will denote the set of equivalence classes of $S$ on $A$.

In this paper $\succsim$ will always denote a binary relation on a set $X=\prod_{i=1}^{n} X_{i}$ with $n \geq 2$. Elements of $X$ will be interpreted as alternatives evaluated on a set $N=\{1,2, \ldots, n\}$ of attributes and $\succsim$ as a "large preference relation" ( $x \succsim y$ being read as " $x$ is at least as good as $y$ ") between these alternatives. We note $\succ($ resp. $\sim)$ the asymmetric (resp. symmetric) part of $\succsim$. A similar convention holds when $\succsim$ is starred, superscripted and/or subscripted.

For any nonempty subset $J$ of the set of attributes $N$, we denote by $X_{J}$ (resp. $X_{-J}$ ) the set $\prod_{i \in J} X_{i}$ (resp. $\prod_{i \notin J} X_{i}$ ). With customary abuse of notation, $\left(x_{J}, y_{-J}\right)$ will denote the element $w \in X$ such that $w_{i}=x_{i}$ if $i \in J$ and $w_{i}=y_{i}$ otherwise. When $J=\{i\}$ we shall simply write $X_{-i}$ and $\left(x_{i}, y_{-i}\right)$.

Let $J$ be a nonempty set of attributes. We define the following binary relations on $X_{J}$ :

$$
\begin{gathered}
x_{J} \succsim_{J} y_{J} \text { iff }\left(x_{J}, z_{-J}\right) \succsim\left(y_{J}, z_{-J}\right), \text { for all } z_{-J} \in X_{-J}, \\
x_{J} \succsim_{J}^{\circ} y_{J} \text { iff }\left(x_{J}, z_{-J}\right) \succsim\left(y_{J}, z_{-J}\right), \text { for some } z_{-J} \in X_{-J},
\end{gathered}
$$

where $x_{J}, y_{J} \in X_{J}$. When $J=\{i\}$ we write $\succsim_{i}$ instead of $\succsim_{\{i\}}$.

If, for all $x_{J}, y_{J} \in X_{J}, x_{J} \succsim_{J}^{\circ} y_{J}$ implies $x_{J} \succsim_{J} y_{J}$, we say that $\succsim$ is independent for $J$. If $\succsim$ is independent for all nonempty subsets of attributes we say that $\succsim$ is independent. It is not difficult to see that a binary relation is independent if and only if it is independent for $N \backslash\{i\}$, for all $i \in N$ (Wakker, 1989). A relation is said to be weakly independent if it is independent for all subsets containing a single attribute; while independence implies weak independence, it is clear that the converse is not true (Wakker, 1989). 


\section{Nontransitive Decomposable Models}

In view of the discussion in section 1 , the most general model we envisage here is such that:

$$
x \succsim y \Leftrightarrow F\left(p_{1}\left(x_{1}, y_{1}\right), p_{2}\left(x_{2}, y_{2}\right), \ldots, p_{n}\left(x_{n}, y_{n}\right)\right) \geq 0
$$

where $p_{i}$ are real-valued functions on $X_{i}^{2}$ and $F$ is a real-valued function on $\prod_{i=1}^{n} p_{i}\left(X_{i}^{2}\right)$, i.e. the Cartesian product of the codomains of the functions $p_{i}$.

As already noted by Goldstein (1991) all binary relations satisfy model (M) when $X$ is finite or countably infinite (see section 4). In particular, it should be noticed that model $(\mathrm{M})$ does not even imply the reflexivity of $\succsim$ which seems a hardly disputable property of any large preference relation. Requiring (M) together with reflexivity leads to a model that is not much constrained however.

In order to further specify model (M), it is useful to consider the additive nontransitive model (4). Like model (M), it does not imply the reflexivity of $\succsim$ unless some additional constraints are imposed on the functions $p_{i}$. A simple way to obtain the reflexivity of $\succsim$ in model (4) is to impose that $p_{i}\left(x_{i}, x_{i}\right)=0$ (Vind, 1991). This also entails that $\succsim$ is independent. Mimicking this additional condition in model (M) leads to model (M0) in which:

$$
x \succsim y \Leftrightarrow F\left(p_{1}\left(x_{1}, y_{1}\right), p_{2}\left(x_{2}, y_{2}\right), \ldots, p_{n}\left(x_{n}, y_{n}\right)\right) \geq 0
$$

with $p_{i}\left(x_{i}, x_{i}\right)=0$, for all $x_{i} \in X_{i}$ and all $i \in N$, and $F(\mathbf{0}) \geq 0$, abusing notations in an obvious way.

Keeping in mind the additive nontransitive model (4), it is natural to add to model (M0) the additional requirement that $F$ is nondecreasing ${ }^{1}$ or increasing in all its arguments. This respectively leads to models (M1) and $\left(\mathrm{M} 1^{\prime}\right)$

Except when $x_{i}=y_{i}$, models (M1) and (M1') do not tie together the values of $p_{i}\left(x_{i}, y_{i}\right)$ and $p_{i}\left(y_{i}, x_{i}\right)$. Following what has often been done with model (4) (Fishburn, 1990b, 1991b), a simple way to establish such a link is to impose the skew symmetry of each function $p_{i}$, i.e. $p_{i}\left(x_{i}, y_{i}\right)=-p_{i}\left(y_{i}, x_{i}\right)$, for all $x_{i}, y_{i} \in X_{i}$. Adding this condition to (M1) and (M1') leads to (M2)

\footnotetext{
${ }^{1}$ Let $f$ be a real-valued function on $K \subseteq \mathbb{R}^{k}$. We say that $f$ is nondecreasing (resp. increasing) in its $j$ th argument if, for all $x \in K$ and all $y_{j} \in X_{j}$ such that $\left(y_{j}, x_{-j}\right) \in$ $K,\left[y_{j}>x_{j}\right] \Rightarrow\left[f\left(y_{j}, x_{-j}\right) \geq f(x)\right]$ (resp. $>$ ). Furthermore, when $K$ is such that $x \in K$ $\Rightarrow-x \in K$, we say that $f$ is odd if, for all $x \in K, f(x)=-f(-x)$.
} 
Table 1: Definition of the various models studied

$\begin{array}{cc}\text { (M) } & x \succsim y \Leftrightarrow F\left(p_{1}\left(x_{1}, y_{1}\right), p_{2}\left(x_{2}, y_{2}\right), \ldots, p_{n}\left(x_{n}, y_{n}\right)\right) \geq 0 \\ \text { (M0) } & (\mathrm{M}) \text { with } p_{i}\left(x_{i}, x_{i}\right)=0 \text { and } F(\mathbf{0}) \geq 0 \\ \text { (M1) } & (\mathrm{M} 0) \text { with } F \text { nondecreasing in all its arguments } \\ \left(\mathrm{M} 1^{\prime}\right) & (\mathrm{M} 0) \text { with } F \text { increasing in all its arguments } \\ (\mathrm{M} 2) & (\mathrm{M} 1) \text { with } p_{i} \text { skew symmetric } \\ \left(\mathrm{M}^{\prime}\right) & \left(\mathrm{M}^{\prime}\right) \text { with } p_{i} \text { skew symmetric } \\ (\mathrm{M} 3) & (\mathrm{M} 2) \text { with } F \text { odd } \\ \left.\text { (M3 }^{\prime}\right) & \left(\mathrm{M} 2^{\prime}\right) \text { with } F \text { odd }\end{array}$

and $\left(\mathrm{M} 2^{\prime}\right)$. Finally, in order to bring $F$ even closer to an addition operation, we may add to models (M2) and (M2') the requirement that $F$ should be odd; this defines models (M3) and (M3').

These various models combine in different ways the increasingness of $F$, its oddness and the skew symmetry of the functions $p_{i}$; some of them will turn out to be equivalent. The definition of these various models is summarized in table 1.

It should be noticed that we do not study here all possible combinations of the various properties added to $(\mathrm{M})$, e.g. we do not consider a model in which $F$ would be odd but the $p_{i}$ would not necessarily be skew symmetric. Similarly, other restrictions on $F$ and $p_{i}$ could be considered (e.g. $p_{i}\left(x_{i}, y_{i}\right) \times$ $\left.p_{i}\left(y_{i}, x_{i}\right) \leq 0\right)$. Most of the models that have been left aside are either easily analyzed using our conditions or trivial.

All the models presented in table 1 involve some form of "inter-attribute decomposability" that we shall study in section 4. Models (M1) and (M1') were introduced by Goldstein (1991) under the name "decomposable thresholds models". It is clear that:

- $\left(\mathrm{M}^{\prime}\right) \Rightarrow(\mathrm{M} 3),\left(\mathrm{M}^{\prime}\right) \Rightarrow(\mathrm{M} 2),\left(\mathrm{M}^{\prime}\right) \Rightarrow(\mathrm{M} 1)$,

- $\left(\mathrm{M}^{\prime}\right) \Rightarrow\left(\mathrm{M}^{\prime}\right) \Rightarrow\left(\mathrm{M}^{\prime}\right)$,

- $(\mathrm{M} 3) \Rightarrow(\mathrm{M} 2) \Rightarrow(\mathrm{M} 1)$.

The nontransitivity and/or noncompleteness of $\succsim$ may obscure some features of our models. We sum up a few useful observations in the following proposition. 
Proposition 1 Let $\succsim$ be a binary relation on $X=\prod_{i=1}^{n} X_{i}$ and $J \subseteq N$.

1. If $\succsim$ satisfies model (MO) then it is reflexive and independent.

2. If $\succsim$ satisfies model (M1) or (M1') then: $\left[x_{i} \succ_{i} y_{i}\right.$ for all $\left.i \in J \subseteq N\right] \Rightarrow$ $\operatorname{Not}\left[\left(y_{J} \succsim_{J} x_{J}\right)\right]$.

3. If $\succsim$ satisfies model (M2) or (M2') then:

- ${ }_{i}$ is complete,

- $\left[x_{i} \succ_{i} y_{i}\right.$ for all $\left.i \in J \subseteq N\right] \Rightarrow\left[x_{J} \succ_{J} y_{J}\right]$.

4. If $\succsim$ satisfies model (M3) then it is complete.

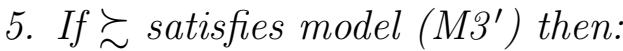

- $\left[x_{i} \succsim_{i} y_{i}\right.$ for all $\left.i \in J \subseteq N\right] \Rightarrow\left[x_{J} \succsim_{J} y_{J}\right]$,

- $\left[x_{i} \succsim_{i} y_{i}\right.$ for all $i \in J \subseteq N, x_{j} \succ_{j} y_{j}$, for some $\left.j \in J\right] \Rightarrow\left[x_{J} \succ_{J}\right.$ $\left.y_{J}\right]$.

\section{Proof of Proposition 1}

1) Obvious since $p_{i}\left(x_{i}, x_{i}\right)=0$ and $F(\mathbf{0}) \geq 0$.

2) Using obvious notations, $x_{i} \succ_{i} y_{i}$ implies $\operatorname{Not}\left[y_{i} \succsim_{i} x_{i}\right]$ so that $F\left(p_{i}\left(y_{i}\right.\right.$, $\left.\left.x_{i}\right), \mathbf{0}\right)<0$. Since $F(\mathbf{0}) \geq 0$ we know that $p_{i}\left(y_{i}, x_{i}\right)<0$ using the nondecreasingness of $F$. Suppose now that $y_{J} \succsim_{J} x_{J}$ so that $F\left(\left(p_{i}\left(y_{i}, x_{i}\right)_{i \in J}\right), \mathbf{0}\right) \geq 0$. Since $p_{i}\left(y_{i}, x_{i}\right)<0$ for all $i \in J$ and $F$ is nondecreasing, this leads to $F\left(p_{j}\left(y_{j}, x_{j}\right), \mathbf{0}\right) \geq 0$ for any $j \in J$, a contradiction.

3) $\operatorname{Not}\left[x_{i} \succsim_{i} y_{i}\right]$ and $\operatorname{Not}\left[y_{i} \succsim_{i} x_{i}\right]$ imply $F\left(p_{i}\left(x_{i}, y_{i}\right), \mathbf{0}\right)<0$ and $F\left(p_{i}\left(y_{i}\right.\right.$, $\left.\left.x_{i}\right), \mathbf{0}\right)<0$. Since $F(\mathbf{0}) \geq 0$ and $F$ is nondecreasing, we have $p_{i}\left(x_{i}, y_{i}\right)<0$ and $p_{i}\left(y_{i}, x_{i}\right)<0$, which contradicts the skew symmetry of $p_{i}$. Hence $\succsim_{i}$ is complete. Observe that $x_{i} \succ_{i} y_{i}$ is equivalent to $F\left(p_{i}\left(x_{i}, y_{i}\right), \mathbf{0}\right) \geq 0$ and $F\left(p_{i}\left(y_{i}, x_{i}\right), \mathbf{0}\right)<0$. Since $F(\mathbf{0}) \geq 0$ we know that $p_{i}\left(y_{i}, x_{i}\right)<0$ using the nondecreasingness of $F$. The skew symmetry of $p_{i}$ implies $p_{i}\left(x_{i}, y_{i}\right)>0>$ $p_{i}\left(y_{i}, x_{i}\right)$ and the desired property easily follows using the nondecreasingness of $F$.

4) Obvious from the skew symmetry of $p_{i}$ and the oddness of $F$.

5) Since $F$ is increasing and odd, we have $x_{i} \succsim_{i} y_{i} \Leftrightarrow p_{i}\left(x_{i}, y_{i}\right) \geq 0$. The desired properties easily follow from the increasingness of $F$ and $F(\mathbf{0})=0$. 
Except for $\left(\mathrm{M}^{\prime}\right)$, the monotonicity properties of our models linking $\succsim$ and $\succsim_{i}$ may seem disappointing. Such properties should however be analyzed keeping in mind that we are dealing with possibly nontransitive and/or noncomplete preferences. In such a framework, some "obvious properties" may not always be desirable. For example, when the relations $\sim_{i}$ are not transitive, it may not be reasonable to impose that:

$$
\left[x_{i} \sim_{i} y_{i} \text { for all } i \in J\right] \Rightarrow\left[x_{J} \sim_{J} y_{J}\right]
$$

which would forbid any interaction between separately non-noticeable differences on each attribute (on this point see Gilboa and Lapson (1995) or Pirlot and Vincke (1997)). Furthermore, as we shall see in section 4, nice monotonicity properties obtain when "preference differences" are adequately modelled on each attribute (see lemma 3 ).

\section{Results}

\subsection{Axioms}

This section studies the variety of nontransitive decomposable models introduced in section 3. The intuition behind these models is that the functions $p_{i}$ "measure" preference differences between elements of $X_{i}$, these differences being aggregated using $F$.

Wakker $(1988,1989)$ has powerfully shown how the consideration of induced relations comparing "preference differences" on each attribute may illuminate the analysis of conjoint measurement models. We follow the same path. Notice however that, although we use similar notation, our definitions of relations comparing preference difference differ from his because of the absence of structural assumptions on $X$.

Given a binary relation $\succsim$ on $X$, we define the binary relation $\succsim_{i}^{*}$ on $X_{i}^{2}$ letting, for all $x_{i}, y_{i}, z_{i}, w_{i} \in X_{i}$,

$$
\begin{aligned}
& \left(x_{i}, y_{i}\right) \succsim_{i}^{*}\left(z_{i}, w_{i}\right) \text { iff } \\
& \quad\left[\text { for all } a_{-i}, b_{-i} \in X_{-i},\left(z_{i}, a_{-i}\right) \succsim\left(w_{i}, b_{-i}\right) \Rightarrow\left(x_{i}, a_{-i}\right) \succsim\left(y_{i}, b_{-i}\right)\right] .
\end{aligned}
$$

Intuitively, if $\left(x_{i}, y_{i}\right) \succsim_{i}^{*}\left(z_{i}, w_{i}\right)$, it seems reasonable to conclude that the preference difference between $x_{i}$ and $y_{i}$ is not smaller that the preference difference between $z_{i}$ and $w_{i}$. Notice that, by construction, $\succsim_{i}^{*}$ is transitive. 
Contrary to our intuition concerning preference differences, the definition of $\succsim_{i}^{*}$ does not imply that the two "opposite" differences $\left(x_{i}, y_{i}\right)$ and $\left(y_{i}, x_{i}\right)$ are linked. Henceforth we introduce the binary relation $\succsim_{i}^{* *}$ on $X_{i}^{2}$ letting, for all $x_{i}, y_{i}, z_{i}, w_{i} \in X_{i}$,

$$
\left(x_{i}, y_{i}\right) \succsim_{i}^{* *}\left(z_{i}, w_{i}\right) \text { iff }\left[\left(x_{i}, y_{i}\right) \succsim_{i}^{*}\left(z_{i}, w_{i}\right) \text { and }\left(w_{i}, z_{i}\right) \succsim_{i}^{*}\left(y_{i}, x_{i}\right)\right] \text {. }
$$

It is easy to see that $\succsim_{i}^{* *}$ is transitive and reversible, i.e. $\left(x_{i}, y_{i}\right) \succsim_{i}^{* *}\left(z_{i}, w_{i}\right) \Leftrightarrow$ $\left(w_{i}, z_{i}\right) \succsim_{i}^{* *}\left(y_{i}, x_{i}\right)$.

The relations $\succsim_{i}^{*}$ and $\succsim_{i}^{* *}$ both appear to capture the idea of comparison of preference differences between elements of $X_{i}$ induced by the relation $\succsim$. Hence, they are good candidates to serve as the basis of the definition of the functions $p_{i}$. They will not serve well this purpose however unless they are complete. Hence, the introduction of the following two conditions.

Let $\succsim$ be a binary relation on a set $X=\prod_{i=1}^{n} X_{i}$. For $i \in N$, this relation is said to satisfy:

$R C 1_{i}$ if

$$
\left.\begin{array}{c}
\left(x_{i}, a_{-i}\right) \succsim\left(y_{i}, b_{-i}\right) \\
\text { and } \\
\left(z_{i}, c_{-i}\right) \succsim\left(w_{i}, d_{-i}\right)
\end{array}\right\} \Rightarrow\left\{\begin{array}{l}
\left(x_{i}, c_{-i}\right) \succsim\left(y_{i}, d_{-i}\right) \\
\text { or } \\
\left(z_{i}, a_{-i}\right) \succsim\left(w_{i}, b_{-i}\right),
\end{array}\right.
$$

$R C 2_{i}$ if

$$
\left.\begin{array}{c}
\left(x_{i}, a_{-i}\right) \succsim\left(y_{i}, b_{-i}\right) \\
\text { and } \\
\left(y_{i}, c_{-i}\right) \succsim\left(x_{i}, d_{-i}\right)
\end{array}\right\} \Rightarrow\left\{\begin{array}{l}
\left(z_{i}, a_{-i}\right) \succsim\left(w_{i}, b_{-i}\right) \\
\text { or } \\
\left(w_{i}, c_{-i}\right) \succsim\left(z_{i}, d_{-i}\right),
\end{array}\right.
$$

for all $x_{i}, y_{i}, z_{i}, w_{i} \in X_{i}$ and all $a_{-i}, b_{-i}, c_{-i}, d_{-i} \in X_{-i}$. We say that $\succsim$ satisfies $R C 1$ (resp. $R C 2$ ) if it satisfies $R C 1_{i}$ (resp. $R C 2_{i}$ ) for all $i \in N$.

Condition $R C 1_{i}$ implies that any two ordered pairs $\left(x_{i}, y_{i}\right)$ and $\left(z_{i}, w_{i}\right)$ of elements of $X_{i}$ are comparable in terms of the relation $\succsim_{i}^{*}$. Indeed, it is easy to see that supposing $\operatorname{Not}\left[\left(x_{i}, y_{i}\right) \succsim_{i}^{*}\left(z_{i}, w_{i}\right)\right]$ and $\operatorname{Not}\left[\left(z_{i}, w_{i}\right) \succsim_{i}^{*}\left(x_{i}, y_{i}\right)\right]$ leads to a violation of $R C 1_{i}$. Similarly, $R C 2_{i}$ implies that the two opposite differences $\left(x_{i}, y_{i}\right)$ and $\left(y_{i}, x_{i}\right)$ are linked. In terms of the relation $\succsim_{i}^{*}$, it says that if the preference difference between $x_{i}$ and $y_{i}$ is not at least as large as the preference difference between $z_{i}$ and $w_{i}$ then the preference difference between $y_{i}$ and $x_{i}$ should be at least as large as the preference difference between $w_{i}$ and $z_{i}$.

We summarize these observations in the following lemma; we omit its straightforward proof. 
Lemma 1 We have:

1. $\left[\succsim_{i}^{*}\right.$ is complete $] \Leftrightarrow R C 1_{i}$,

2. $R C 2_{i} \Leftrightarrow$

$\left[\right.$ for all $\left.x_{i}, y_{i}, z_{i}, w_{i} \in X_{i}, \operatorname{Not}\left[\left(x_{i}, y_{i}\right) \succsim_{i}^{*}\left(z_{i}, w_{i}\right)\right] \Rightarrow\left(y_{i}, x_{i}\right) \succsim_{i}^{*}\left(w_{i}, z_{i}\right)\right]$,

3. $\left[\succsim_{i}^{* *}\right.$ is complete $] \Leftrightarrow\left[R C 1_{i}\right.$ and $\left.R C 2_{i}\right]$.

Condition $R C 1$ was introduced in Bouyssou (1986) under the name "weak cancellation". Technically $R C 1_{i}$ amounts to defining a biorder, in the sense of Ducamp and Falmagne (1969) and Doignon, Ducamp, and Falmagne (1984), between the sets $X_{i}^{2}$ and $X_{-i}^{2}$. The extension of condition $R C 1$ to subsets of attributes is central to the analysis of (4) with $p_{i}\left(x_{i}, x_{i}\right)=0$ by Vind (1991) where this condition is called independence. Condition $R C 2$ seems to be new.

We say that $\succsim$ satisfies:

$T C_{i}$ if

$$
\left.\begin{array}{l}
\left(x_{i}, a_{-i}\right) \succsim\left(y_{i}, b_{-i}\right) \\
\text { and } \\
\left(z_{i}, b_{-i}\right) \succsim\left(w_{i}, a_{-i}\right) \\
\text { and } \\
\left(w_{i}, c_{-i}\right) \succsim\left(z_{i}, d_{-i}\right)
\end{array}\right\} \Rightarrow\left(x_{i}, c_{-i}\right) \succsim\left(y_{i}, d_{-i}\right),
$$

for all $x_{i}, y_{i}, z_{i}, w_{i} \in X_{i}$ and all $a_{-i}, b_{-i}, c_{-i}, d_{-i} \in X_{-i}$. We say that $\succsim$ satisfies $T C$ if it satisfies $T C_{i}$ for all $i \in N$.

Condition $T C_{i}$ (Triple Cancellation) is a classical cancellation condition that has been often used in the analysis of model (1) (Krantz et al., 1971; Wakker, 1989). As shown below, it implies both $R C 1$ and $R C 2$ when $\succsim$ is complete. We refer to Wakker $(1988,1989)$ for a detailed analysis of $T C$ including its interpretation in terms of difference of preference.

The following lemma (that omits the implications directly resulting from the links between our various models) shows that $R C 1, R C 2$ and $T C$ are implied by various versions of our nontransitive decomposable models and states some links between these conditions.

Lemma 2 We have:

1. Model (M1) implies RC1,

2. Model (M2) implies RC2, 


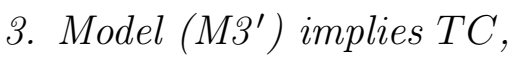

4. If $\succsim$ is complete, $T C_{i}$ implies $R C 1_{i}$ and $R C 2_{i}$,

5. If $\succsim$ satisfies $R C 2$ then it is independent and either reflexive or irreflexive,

6. Reflexivity, independence and $R C 1$ are independent conditions,

7. In the class of complete relations, $R C 1$ and $R C 2$ are independent conditions.

\section{Proof of Lemma 2}

1) Suppose that $\left(x_{i}, a_{-i}\right) \succsim\left(y_{i}, b_{-i}\right)$ and $\left(z_{i}, c_{-i}\right) \succsim\left(w_{i}, d_{-i}\right)$. Using model (M1) we have:

$$
F\left(p_{i}\left(x_{i}, y_{i}\right),\left(p_{j}\left(a_{j}, b_{j}\right)\right)_{j \neq i}\right) \geq 0
$$

and

$$
F\left(p_{i}\left(z_{i}, w_{i}\right),\left(p_{j}\left(c_{j}, d_{j}\right)\right)_{j \neq i}\right) \geq 0,
$$

abusing notations in an obvious way.

If $p_{i}\left(x_{i}, y_{i}\right) \geq p_{i}\left(z_{i}, w_{i}\right)$ then using the nondecreasingness of $F$, we have $F\left(p_{i}\left(x_{i}, y_{i}\right),\left(p_{j}\left(c_{j}, d_{j}\right)\right)_{j \neq i}\right) \geq 0$ so that $\left(x_{i}, c_{-i}\right) \succsim\left(y_{i}, d_{-i}\right)$. If $p_{i}\left(z_{i}, w_{i}\right)>$ $p_{i}\left(x_{i}, y_{i}\right)$ we have $F\left(p_{i}\left(z_{i}, w_{i}\right),\left(p_{j}\left(a_{j}, b_{j}\right)\right)_{j \neq i}\right) \geq 0$ so that $\left(z_{i}, a_{-i}\right) \succsim\left(w_{i}, b_{-i}\right)$.

2) Suppose that $\left(x_{i}, a_{-i}\right) \succsim\left(y_{i}, b_{-i}\right)$ and $\left(y_{i}, c_{-i}\right) \succsim\left(x_{i}, d_{-i}\right)$. We thus have:

$$
F\left(p_{i}\left(x_{i}, y_{i}\right),\left(p_{j}\left(a_{j}, b_{j}\right)\right)_{j \neq i}\right) \geq 0
$$

and

$$
F\left(p_{i}\left(y_{i}, x_{i}\right),\left(p_{j}\left(c_{j}, d_{j}\right)\right)_{j \neq i}\right) \geq 0 .
$$

If $p_{i}\left(x_{i}, y_{i}\right) \geq p_{i}\left(z_{i}, w_{i}\right)$, the skew symmetry of $p_{i}$ implies $p_{i}\left(w_{i}, z_{i}\right) \geq p_{i}\left(y_{i}, x_{i}\right)$ so that $\left(w_{i}, c_{-i}\right) \succsim\left(z_{i}, d_{-i}\right)$ using the nondecreasingness of $F$. Similarly, if $p_{i}\left(z_{i}, w_{i}\right)>p_{i}\left(x_{i}, y_{i}\right)$ we have, using the nondecreasingness of $F,\left(z_{i}, a_{-i}\right) \succsim$ $\left(w_{i}, b_{-i}\right)$.

3) Suppose that $\left(x_{i}, a_{-i}\right) \succsim\left(y_{i}, b_{-i}\right),\left(z_{i}, b_{-i}\right) \succsim\left(w_{i}, a_{-i}\right),\left(w_{i}, c_{-i}\right) \succsim\left(z_{i}\right.$, $\left.d_{-i}\right)$ and $\operatorname{Not}\left[\left(x_{i}, c_{-i}\right) \succsim\left(y_{i}, d_{-i}\right)\right]$. Using $\left(\mathrm{M}^{\prime}\right)$ we know that:

$$
\begin{aligned}
& F\left(p_{i}\left(x_{i}, y_{i}\right),\left(p_{j}\left(a_{j}, b_{j}\right)\right)_{j \neq i}\right) \geq 0 \\
& F\left(p_{i}\left(z_{i}, w_{i}\right),\left(p_{j}\left(b_{j}, a_{j}\right)\right)_{j \neq i}\right) \geq 0
\end{aligned}
$$




$$
F\left(p_{i}\left(w_{i}, z_{i}\right),\left(p_{j}\left(c_{j}, d_{j}\right)\right)_{j \neq i}\right) \geq 0
$$

and

$$
F\left(p_{i}\left(x_{i}, y_{i}\right),\left(p_{j}\left(c_{j}, d_{j}\right)\right)_{j \neq i}\right)<0 .
$$

Using the oddness of $F$, its increasingness and the skew symmetry of the $p_{i}$ 's, the first two inequalities imply $p_{i}\left(x_{i}, y_{i}\right) \geq p_{i}\left(w_{i}, z_{i}\right)$ whereas the last two imply that $p_{i}\left(x_{i}, y_{i}\right)<p_{i}\left(w_{i}, z_{i}\right)$, a contradiction.

4) In contradiction with $R C 1_{i}$ suppose that $\left(x_{i}, a_{-i}\right) \succsim\left(y_{i}, b_{-i}\right),\left(z_{i}, c_{-i}\right) \succsim$ $\left(w_{i}, d_{-i}\right), \operatorname{Not}\left[\left(z_{i}, a_{-i}\right) \succsim\left(w_{i}, b_{-i}\right)\right]$ and $\operatorname{Not}\left[\left(x_{i}, c_{-i}\right) \succsim\left(y_{i}, d_{-i}\right)\right]$. Since $\succsim$ is complete, we have $\left(w_{i}, b_{-i}\right) \succ\left(z_{i}, a_{-i}\right)$. Using $T C_{i},\left(x_{i}, a_{-i}\right) \succsim\left(y_{i}, b_{-i}\right)$, $\left(w_{i}, b_{-i}\right) \succ\left(z_{i}, a_{-i}\right)$ and $\left(z_{i}, c_{-i}\right) \succsim\left(w_{i}, d_{-i}\right)$ imply $\left(x_{i}, c_{-i}\right) \succsim\left(y_{i}, d_{-i}\right)$, a contradiction.

Similarly suppose, in contradiction with $R C 2_{i}$ that $\left(x_{i}, a_{-i}\right) \succsim\left(y_{i}, b_{-i}\right)$, $\left(y_{i}, c_{-i}\right) \succsim\left(x_{i}, d_{-i}\right), \operatorname{Not}\left[\left(z_{i}, a_{-i}\right) \succsim\left(w_{i}, b_{-i}\right)\right]$ and $\operatorname{Not}\left[\left(w_{i}, c_{-i}\right) \succsim\left(z_{i}, d_{-i}\right)\right]$. Since $\succsim$ is complete, we know that $\left(w_{i}, b_{-i}\right) \succ\left(z_{i}, a_{-i}\right)$. Using $T C_{i},\left(w_{i}, b_{-i}\right)$ $\succ\left(z_{i}, a_{-i}\right),\left(x_{i}, a_{-i}\right) \succsim\left(y_{i}, b_{-i}\right)$ and $\left(y_{i}, c_{-i}\right) \succsim\left(x_{i}, d_{-i}\right)$ imply $\left(w_{i}, c_{-i}\right) \succsim$ $\left(z_{i}, d_{-i}\right)$, a contradiction.

5) If $\left(x_{i}, a_{-i}\right) \succsim\left(x_{i}, b_{-i}\right), R C 2_{i}$ implies $\left(y_{i}, a_{-i}\right) \succsim\left(y_{i}, b_{-i}\right)$ for all $y_{i} \in X_{i}$ so that $\succsim$ is independent. It is clear that an independent relation is either reflexive or irreflexive.

6) In order to show that these three properties are completely independent, we need $2^{3}=8$ examples. It is easy to build a relation $\succsim$ that does not satisfy $R C 1$ and is neither reflexive nor independent (e.g. take $X=\{a, b\} \times\{z, w\}$ and let $\succsim$ be an empty relation on $X$ except that $(a, z) \succsim(b, z)$ and $(b, w) \succsim(a, w))$. Any relation $\succsim$ satisfying the additive utility model (1) satisfies the three properties. We provide here the six remaining examples.

1. Let $X=\{a, b\} \times\{z, w\}$ and consider $\succsim$ on $X$ defined by: for all $(\alpha, \beta)$, $(\gamma, \delta) \in X,(\alpha, \beta) \succsim(\gamma, \delta) \Leftrightarrow f(\alpha, \gamma)+g(\beta, \delta) \geq 0$, where $f$ and $g$ are such that: $f(a, a)=-1, f(a, b)=0.5, f(b, a)=-0.5, f(b, b)=1$, $g(z, z)=g(w, w)=g(w, z)=1, g(z, w)=0$

It is easy to see that $\succsim$ is reflexive. It satisfies $R C 1$ by construction (see remark 5 in section 4.4$)$. It is not independent since $(b, z) \succsim(b, w)$ and $\operatorname{Not}[(a, z) \succsim(a, w)]$.

2. In example 1 , taking $f(a, a)=-2$ leads to relation $\succsim$ that verifies $R C 1$ but is neither independent nor reflexive (since $\operatorname{Not}[(a, z) \succsim(a, z)])$. 
3. Let $X=\{a, b\} \times\{z, w\}$ and consider $\succsim$ on $X$ defined by: for all $(\alpha, \beta),(\gamma, \delta) \in X,(\alpha, \beta) \succsim(\gamma, \delta) \Leftrightarrow f(\alpha, \gamma)+g(\beta, \delta) \geq 0$, where $f$ and $g$ are such that: $f(a, a)=f(b, b)=f(b, a)=-1, f(a, b)=1$, $g(z, z)=g(w, w)=0, g(z, w)=1, g(w, z)=-1$

It is easy to see that $\succsim$ is not reflexive (it is in fact irreflexive). It satisfies $R C 1$ by construction (see remark 5 in section 4.4). Since $f(a, a)=f(b, b)$ and $g(z, z)=g(w, w), \succsim$ is clearly independent.

4. Let $X=\{a, b, c\} \times\{z, w\}$ and consider $\succsim$ on $X$ that is a clique (with all loops) except that $\operatorname{Not}[(a, z) \succsim(c, w)]$ and $\operatorname{Not}[(a, w) \succsim(b, z)]$.

It is clear that $\succsim$ is reflexive. It can easily be checked that $\succsim$ is independent. It does not satisfy $R C 1$ since: $(a, z) \succsim(b, w),(a, w) \succsim(c, z)$, $\operatorname{Not}[(a, z) \succsim(c, w)]$ and $\operatorname{Not}[(a, w) \succsim(b, z)]$.

5. Modifying example 4 in order to have $\succsim$ irreflexive gives an example of relation that is independent but violates $R C 1$ and reflexivity.

6. Modifying example 4 in order to have $\operatorname{Not}[(b, z) \succsim(b, w)]$ leads to relation $\succsim$ that is reflexive but violates independence and $R C 1$.

7) Any relation $\succsim$ satisfying the additive utility model (1) is complete and satisfies both $R C 1$ and $R C 2$. We provide here the three remaining examples.

1. Let $X=\{a, b, c\} \times\{z, w, k\}$ and consider $\succsim$ on $X$ that is a clique (with all loops) except that $\operatorname{Not}[(a, z) \succsim(c, w)], \operatorname{Not}[(a, k) \succsim(b, z)]$ and $\operatorname{Not}[(c, z) \succsim(a, w)]$. It is clear that $\succsim$ is complete. Since $(a, z) \succsim(b, w)$, $(c, k) \succsim(a, z), \operatorname{Not}[(a, k) \succsim(b, z)]$ and $\operatorname{Not}[(c, z) \succsim(a, w)], \succsim$ violates $R C 1$. Since $(a, z) \succsim(b, w),(b, z) \succsim(a, w), \operatorname{Not}[(a, z) \succsim(c, w)]$ and $\operatorname{Not}[(c, z) \succsim(a, w)], \succsim$ violates $R C 2$.

2. Modify example 1 adding the relation $(a, z) \succsim(c, w)$. It is clear that $\succsim$ is complete and violates $R C 1$. Using lemma 1.(2), it is not difficult to see that it satisfies $R C 2$.

3. Let $X=\{a, b\} \times\{z, w\}$ and consider $\succsim$ on $X$ defined by: for all $(\alpha, \beta)$, $(\gamma, \delta) \in X,(\alpha, \beta) \succsim(\gamma, \delta) \Leftrightarrow f(\alpha, \gamma)+g(\beta, \delta) \geq 0$, where $f$ and $g$ are such that: $f(a, a)=-1, f(a, b)=f(b, a)=f(b, b)=1, g(z, w)=$ $0, g(z, z)=g(w, w)=g(w, z)=1$. 
It is easy to see that $\succsim$ is complete. It satisfies $R C 1$ by construction (see remark 5 in section 4.4). It is not independent since $(b, z) \succsim(b, w)$ and $\operatorname{Not}[(a, z) \succsim(a, w)]$. In view of part 5 of this lemma, this shows that $R C 2$ is violated.

For the sake of easy reference, we note a few useful connections between $\succsim_{i}^{*}, \succsim_{i}^{* *}$ and $\succsim$ in the following lemma.

Lemma 3 For all $x, y \in X$ and all $z_{i}, w_{i} \in X_{i}$,

1. $\left[x \succsim y\right.$ and $\left.\left(z_{i}, w_{i}\right) \succsim_{i}^{*}\left(x_{i}, y_{i}\right)\right] \Rightarrow\left(z_{i}, x_{-i}\right) \succsim\left(w_{i}, y_{-i}\right)$,

2. $\left[\left(z_{i}, w_{i}\right) \sim_{i}^{*}\left(x_{i}, y_{i}\right)\right.$ for all $\left.i \in N\right] \Rightarrow[x \succsim y \Leftrightarrow z \succsim w]$,

3. $\left[x \succ y\right.$ and $\left.\left(z_{i}, w_{i}\right) \succsim_{i}^{* *}\left(x_{i}, y_{i}\right)\right] \Rightarrow\left(z_{i}, x_{-i}\right) \succ\left(w_{i}, y_{-i}\right)$,

4. $\left[\left(z_{i}, w_{i}\right) \sim_{i}^{* *}\left(x_{i}, y_{i}\right)\right.$ for all $\left.i \in N\right] \Rightarrow([x \succsim y \Leftrightarrow z \succsim w]$ and $[x \succ y \Leftrightarrow$ $z \succ w])$

5. If $T C_{i}$ holds and $\succsim$ is complete, $\left[x \succsim y\right.$ and $\left.\left(z_{i}, w_{i}\right) \succ_{i}^{* *}\left(x_{i}, y_{i}\right)\right] \Rightarrow$ $\left(z_{i}, x_{-i}\right) \succ\left(w_{i}, y_{-i}\right)$.

\section{Proof of Lemma 3}

1) Obvious from the definition of $\succsim_{i}^{*}$.

By induction, 2) is immediate from 1).

3) Given 1), we know that $\left(z_{i}, x_{-i}\right) \succsim\left(w_{i}, y_{-i}\right)$. Suppose that $\left(w_{i}, y_{-i}\right)$ $\succsim\left(z_{i}, x_{-i}\right)$. Since $\left(z_{i}, w_{i}\right) \succsim_{i}^{* *}\left(x_{i}, y_{i}\right)$ implies $\left.\left(y_{i}, x_{i}\right) \succsim_{i}^{*}\left(w_{i}, z_{i}\right), 1\right)$ implies $y \succsim x$, a contradiction.

4) Immediate from 2) and 3).

5) Notice that 1) implies $\left(z_{i}, x_{-i}\right) \succsim\left(w_{i}, y_{-i}\right)$. Suppose that $\left(w_{i}, y_{-i}\right) \succsim$ $\left(z_{i}, x_{-i}\right)$. From lemmas 2.(4) and 1.(3), we know that $\succsim_{i}^{* *}$ is complete. We thus have $\left(z_{i}, w_{i}\right) \succ_{i}^{* *}\left(x_{i}, y_{i}\right) \Leftrightarrow \operatorname{Not}\left[\left(x_{i}, y_{i}\right) \succsim_{i}^{* *}\left(z_{i}, w_{i}\right)\right] \Leftrightarrow\left[\operatorname{Not}\left[\left(x_{i}, y_{i}\right) \succsim_{i}^{*}\right.\right.$ $\left.\left(z_{i}, w_{i}\right)\right]$ or $\left.\operatorname{Not}\left[\left(w_{i}, z_{i}\right) \succsim_{i}^{*}\left(y_{i}, x_{i}\right)\right]\right]$. In the first case we know that $\operatorname{Not}\left[\left(x_{i}\right.\right.$, $\left.\left.c_{-i}\right) \succsim\left(y_{i}, d_{-i}\right)\right]$ and $\left(z_{i}, c_{-i}\right) \succsim\left(w_{i}, d_{-i}\right)$ for some $c_{-i}, d_{-i} \in X_{-i}$. Using $T C_{i}$ we know that $x \succsim y,\left(w_{i}, y_{-i}\right) \succsim\left(z_{i}, x_{-i}\right)$ and $\left(z_{i}, c_{-i}\right) \succsim\left(w_{i}, d_{-i}\right)$ imply $\left(x_{i}, c_{-i}\right) \succsim\left(y_{i}, d_{-i}\right)$, a contradiction. The other case is similar. 


\subsection{The denumerable case}

Our first result says that for finite or countably infinite sets $X$ conditions $R C 1, R C 2$ and $T C$ combined with reflexivity, independence and/or completeness allow us to characterize our various models.

Theorem 1 Let $\succsim$ be a binary relation on a finite or countably infinite set $X=\prod_{i=1}^{n} X_{i}$. Then:

1. ¿ satisfies model (M),

2. ¿ satisfies model (MO) iff it is reflexive and independent,

3. ¿ satisfies model $\left(M 1^{\prime}\right)$ iff it is reflexive, independent and satisfies $R C 1$,

4. $\succsim$ satisfies model (M2') iff it is reflexive and satisfies $R C 1$ and $R C 2$,

5. $\succsim$ satisfies model (M3) iff it is complete and satisfies RC1 and RC2,

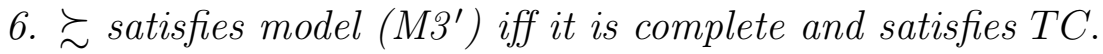

\section{Proof of Theorem 1}

1) Following Goldstein (1991), define on each $X_{i}^{2}$ a binary relation $\sim_{i}^{*}$ letting, for all $x_{i}, y_{i}, z_{i}, w_{i} \in X_{i}:\left(x_{i}, y_{i}\right) \sim_{i}^{*}\left(z_{i}, w_{i}\right)$ iff [for all $a_{-i}, b_{-i} \in$ $\left.X_{-i},\left(z_{i}, a_{-i}\right) \succsim\left(w_{i}, b_{-i}\right) \Leftrightarrow\left(x_{i}, a_{-i}\right) \succsim\left(y_{i}, b_{-i}\right)\right]$. It is easily seen that $\sim_{i}^{*}$ is an equivalence. Since $X$ is finite or countably infinite, there is a real-valued function $p_{i}$ on $X_{i}^{2}$ such that, for all $x_{i}, y_{i}, z_{i}, w_{i} \in X_{i}:\left(x_{i}, y_{i}\right) \sim_{i}^{*}\left(z_{i}, w_{i}\right)$ iff $p_{i}\left(x_{i}, y_{i}\right)=p_{i}\left(z_{i}, w_{i}\right)$. Define $F$ on $\prod_{i=1}^{n} p_{i}\left(X_{i}^{2}\right)$ letting:

$$
F\left(p_{1}\left(x_{1}, y_{1}\right), p_{2}\left(x_{2}, y_{2}\right), \ldots, p_{n}\left(x_{n}, y_{n}\right)\right)=\left\{\begin{array}{l}
1 \text { if } x \succsim y \\
-1 \text { otherwise }
\end{array}\right.
$$

Using the definition of $\sim_{i}^{*}$, it is easy to show that $F$ is well-defined.

Necessity of parts 2 to 6 results from proposition 1.(1) and 1.(4), lemma 2.(1), 2.(2) and 2.(3) and the implications between the various models. We establish sufficiency below.

2) It is clear that: [ $\succsim$ is independent] $\Leftrightarrow$ [for all $i \in N, \succsim$ is independent for $N \backslash\{i\}] \Leftrightarrow\left[\left(x_{i}, x_{i}\right) \sim_{i}^{*}\left(y_{i}, y_{i}\right)\right.$, for all $i \in N$ and all $\left.x_{i}, y_{i} \in X_{i}\right]$.

Since $\succsim$ is independent, we know that all the elements of the diagonal of $X_{i}^{2}$ belong to the same equivalence class of $\sim_{i}^{*}$. Define the functions 
$p_{i}$ as in 1). They can always be chosen so that, for all $i \in N$ and all $x_{i} \in X_{i}, p_{i}\left(x_{i}, x_{i}\right)=0$. Using such functions, define $F$ as in 1$)$. The welldefinedness of $F$ results from the definition of $\sim_{i}^{*}$. Since $\succsim$ is reflexive, we have $F(\mathbf{0})=1 \geq 0$, as required by model (M0).

3) Since $R C 1_{i}$ holds, we know from lemma 1.(1) that $\succsim_{i}^{*}$ is complete and, thus, is a weak order. Since $X$ is finite or countably infinite, there is a real-valued function $p_{i}$ on $X_{i}^{2}$ such that, for all $x_{i}, y_{i}, z_{i}, w_{i} \in X_{i},\left(x_{i}, y_{i}\right) \succsim_{i}^{*}$ $\left(z_{i}, w_{i}\right) \Leftrightarrow p_{i}\left(x_{i}, y_{i}\right) \geq p_{i}\left(z_{i}, w_{i}\right)$. Since $\succsim$ is independent we proceed as in 2) and choose the functions $p_{i}$ so that $p_{i}\left(x_{i}, x_{i}\right)=0$. Given such a particular numerical representation $p_{i}$ of $\succsim_{i}^{*}$ for $i=1,2, \ldots, n$, we define $F$ as follows:

$$
\begin{aligned}
& F\left(p_{1}\left(x_{1}, y_{1}\right), p_{2}\left(x_{2}, y_{2}\right), \ldots, p_{n}\left(x_{n}, y_{n}\right)\right)= \\
& \qquad\left\{\begin{array}{l}
f\left(g\left(p_{1}\left(x_{1}, y_{1}\right), p_{2}\left(x_{2}, y_{2}\right), \ldots, p_{n}\left(x_{n}, y_{n}\right)\right)\right) \text { if } x \succsim y, \\
-f\left(-g\left(p_{1}\left(x_{1}, y_{1}\right), p_{2}\left(x_{2}, y_{2}\right), \ldots, p_{n}\left(x_{n}, y_{n}\right)\right)\right) \text { otherwise },
\end{array}\right.
\end{aligned}
$$

where $g$ is any function from $\mathbb{R}^{n}$ to $\mathbb{R}$ increasing in all its arguments (e.g. $\Sigma)$ and $f$ is any increasing function from $\mathbb{R}$ into $(0,+\infty)$ (e.g. $\exp (\cdot)$ or $\left.\arctan (\cdot)+\frac{\pi}{2}\right)$.

Let us show that $F$ is well-defined and increasing in all its arguments. The well-definedness of $F$ follows from lemma 3.(2) and the definition of the $p_{i}$ 's. To show that $F$ is increasing, suppose that $p_{i}\left(z_{i}, w_{i}\right)>p_{i}\left(x_{i}, y_{i}\right)$, i.e. that $\left(z_{i}, w_{i}\right) \succ_{i}^{*}\left(x_{i}, y_{i}\right)$. If $x \succsim y$, we know from lemma 3.(1) that $\left(z_{i}, x_{-i}\right) \succsim\left(w_{i}, y_{-i}\right)$ and the conclusion follows from the definition of $F$. If $\operatorname{Not}[x \succsim y]$ we have either $\operatorname{Not}\left[\left(z_{i}, x_{-i}\right) \succsim\left(w_{i}, y_{-i}\right)\right]$ or $\left(z_{i}, x_{-i}\right) \succsim\left(w_{i}, y_{-i}\right)$. In either case, the conclusion follows from the definition of $F$. Since $\succsim$ is reflexive, we have $F(\mathbf{0}) \geq 0$, as required.

4) Since $R C 1_{i}$ and $R C 2_{i}$ hold, we know from lemma 1.(3) that $\succsim_{i}^{* *}$ is complete so that it is a weak order. This implies that $\succsim_{i}^{*}$ is a weak order and, since $X$ is finite or countably infinite, there is a real-valued function $q_{i}$ on $X_{i}^{2}$ such that, for all $x_{i}, y_{i}, z_{i}, w_{i} \in X_{i},\left(x_{i}, y_{i}\right) \succsim_{i}^{*}\left(z_{i}, w_{i}\right) \Leftrightarrow q_{i}\left(x_{i}, y_{i}\right) \geq$ $q_{i}\left(z_{i}, w_{i}\right)$. Given a particular numerical representation $q_{i}$ of $\succsim_{i}^{*}$, let $p_{i}\left(x_{i}, y_{i}\right)=$ $q_{i}\left(x_{i}, y_{i}\right)-q_{i}\left(y_{i}, x_{i}\right)$. It is obvious that $p_{i}$ is skew symmetric and represents $\succsim_{i}^{* *}$. Define $F$ as in 3). Its well-definedness and increasingness is proved as in 3). Since $\succsim$ is reflexive, we have $F(\mathbf{0}) \geq 0$, as required.

5) Define $p_{i}$ as in 4) and $F$ as:

$$
F\left(p_{1}\left(x_{1}, y_{1}\right), p_{2}\left(x_{2}, y_{2}\right), \ldots, p_{n}\left(x_{n}, y_{n}\right)\right)=
$$




$$
\left\{\begin{array}{l}
f\left(g\left(p_{1}\left(x_{1}, y_{1}\right), p_{2}\left(x_{2}, y_{2}\right), \ldots, p_{n}\left(x_{n}, y_{n}\right)\right)\right) \text { if } x \succ y \\
0 \text { if } x \sim y \\
-f\left(-g\left(p_{1}\left(x_{1}, y_{1}\right), p_{2}\left(x_{2}, y_{2}\right), \ldots, p_{n}\left(x_{n}, y_{n}\right)\right)\right) \text { otherwise. }
\end{array}\right.
$$

where $g$ is any function from $\mathbb{R}^{n}$ to $\mathbb{R}$, odd and increasing in all its arguments and $f$ is any increasing function from $\mathbb{R}$ into $(0,+\infty)$. That $F$ is well defined follows from lemma 3.(4). It is odd by construction. The nondecreasingness of $F$ follows from lemma 3.(1) and 3.(3).

6 ) Define $p_{i}$ and $F$ as in 5). The increasingness of $F$ follows from lemma 3.(5).

\subsection{The general case}

In order to attain a generalization of theorem 1 to sets of arbitrary cardinality it should be observed that:

- the weak orders $\succsim_{i}^{*}$ may not have a numerical representation and

- a binary relation may have a representation in (M1) with functions $p_{i}$ failing to satisfy:

$$
\left(x_{i}, y_{i}\right) \succsim_{i}^{*}\left(z_{i}, w_{i}\right) \Leftrightarrow p_{i}\left(x_{i}, y_{i}\right) \geq p_{i}\left(z_{i}, w_{i}\right)
$$

i.e. $p_{i}$ is not necessarily a representation of the weak order $\succsim_{i}^{*}$.

The first point should be no surprise. In order to deal with the second point, suppose that the binary relation $\succsim$ satisfies model (M1). The nondecreasingness property of $F$ clearly implies that:

$$
\left(x_{i}, y_{i}\right) \succ_{i}^{*}\left(z_{i}, w_{i}\right) \Rightarrow p_{i}\left(x_{i}, y_{i}\right)>p_{i}\left(z_{i}, w_{i}\right) .
$$

Using this relation, it is not difficult to show that it is always possible to modify a given numerical representation so that it satisfies (7) in (M1). If (7) is violated, pick a particular element from each equivalence class of $\sim_{i}^{*}$ and modify the function $p_{i}$ so that it is constant (and equal to the value of $p_{i}$ for the element picked) on each equivalence class (this is the idea of "regularization" of a scale used in Roberts (1979)). This defines the function $q_{i}$ on $X_{i}^{2}$. Using (M1), it is easily proved that $\left[\left(x_{i}, y_{i}\right) \sim_{i}^{*}\left(z_{i}, w_{i}\right)\right.$ and $\left.p_{i}\left(x_{i}, y_{i}\right)>p_{i}\left(k_{i}, \ell_{i}\right)>p_{i}\left(z_{i}, w_{i}\right)\right]$ imply $\left(x_{i}, y_{i}\right) \sim_{i}^{*}\left(k_{i}, \ell_{i}\right)$ so that the function 
$q_{i}$ satisfies (7). The function $G$ obtained by restricting the original function $F$ to $\prod_{i=1}^{n} q_{i}\left(X_{i}^{2}\right)$ obviously inherits the nondecreasingness property of $F$. Using $G$ together with the functions $q_{i}$ leads to an alternative numerical representation satisfying model (M1) together with (7). A similar reasoning is easily seen to be true for model ( $\left.\mathrm{M} 1^{\prime}\right)$.

A similar technique can be applied with model (M2). Indeed, if $\succsim$ has a representation in (M2) it has a representation in (M1). Consider this representation in (M1) and modify it as above so that (7) is satisfied. Letting $r_{i}\left(x_{i}, y_{i}\right)=q_{i}\left(x_{i}, y_{i}\right)-q_{i}\left(y_{i}, x_{i}\right)$ we easily obtain that:

$$
\left(x_{i}, y_{i}\right) \succsim_{i}^{* *}\left(z_{i}, w_{i}\right) \Leftrightarrow r_{i}\left(x_{i}, y_{i}\right) \geq r_{i}\left(z_{i}, w_{i}\right)
$$

Using these functions $r_{i}$, defining the function $F$ as in the proof of theorem 1.(5) leads to a representation in model (M2) in which (8) is satisfied. A similar technique can be applied with (M2'), (M3) and (M3').

Similarly, there may exist representations in models (M) and (M0) in which the functions $p_{i}$ do not represent the equivalence relations $\sim_{i}^{*}$. It is easy to see that it is always possible to modify a given representation in models (M) and (M0) so that this is the case.

The preceding observations prove the following lemma which defines what could be called regular representations of our models.

Lemma 4 We have:

1. If $\succsim$ satisfies model (M) (resp. (M0)), it has a representation in model (M) (resp. (MO)) such that $\left(x_{i}, y_{i}\right) \sim_{i}^{*}\left(z_{i}, w_{i}\right) \Leftrightarrow p_{i}\left(x_{i}, y_{i}\right)=p_{i}\left(z_{i}, w_{i}\right)$.

2. If $\succsim$ satisfies model (M1) (resp. $\left(M 1^{\prime}\right)$ ), it has a representation in model (M1) (resp. (M1')) such that $\left(x_{i}, y_{i}\right) \succsim_{i}^{*}\left(z_{i}, w_{i}\right) \Leftrightarrow p_{i}\left(x_{i}, y_{i}\right) \geq$ $p_{i}\left(z_{i}, w_{i}\right)$.

3. If $\succsim$ satisfies model (M2) (resp. (M2'), (M3), (M3')), it has a representation in model (M2) (resp. (M2'), (M3), (M3')), such that $\left(x_{i}, y_{i}\right) \succsim_{i}^{* *}$ $\left(z_{i}, w_{i}\right) \Leftrightarrow p_{i}\left(x_{i}, y_{i}\right) \geq p_{i}\left(z_{i}, w_{i}\right)$.

In view of the preceding lemma, the generalization of theorem 1 to sets of arbitrary cardinality for models (M) and (M0) (resp. models (M1') and $\left.\left(\mathrm{M} 2^{\prime}\right),(\mathrm{M} 3),\left(\mathrm{M}^{\prime}\right)\right)$ is at hand if we impose conditions guaranteeing that the relations $\sim_{i}^{*}\left(\right.$ resp. $\succsim_{i}^{*}$ and $\left.\succsim_{i}^{* *}\right)$ have a numerical representation. 
We first deal with models (M) and (M0). We say that $\succsim$ satisfies condition $C_{i}^{*}$ if there is a one-to-one correspondence between $X_{i}^{2} / \sim_{i}^{*}$ and some subset of $\mathbb{R}$. Condition $C^{*}$ is said to hold when $C_{i}^{*}$ holds for all $i \in N$. It is obvious that $C_{i}^{*}$ is a necessary and sufficient condition for the equivalence $\sim_{i}^{*}$ to have a numerical representation. Thus, $C^{*}$ is a necessary condition for models $(\mathrm{M})$ and $(\mathrm{M} 0)$. In view of the proof of part 1 of theorem 1 , it is clear that $C^{*}$ (resp. $C^{*}$, reflexivity and independence) is also sufficient for model (M) (resp. (M0)). It is worth noting that $C^{*}$ is trivially satisfied when, for all $i \in N$, there exists a one-to-one mapping between $X_{i}$ and some subset of $\mathbb{R}$, which is hardly restrictive if, as is usual, $X_{i}$ is interpreted as a set of levels on the $i$ th attribute.

Let $S$ be a binary relation on a set $A$ and let $B \subseteq A$. Following e.g. Krantz et al. (1971, Chapter 2), we say that $B$ is dense in $A$ for $S$ if, for all $a, b \in A,[a S b$ and $N o t[b S a]] \Rightarrow[a S c$ and $c S b$, for some $c \in B]$. The existence of a finite or countably infinite set $B$ dense in $A$ for $S$ is a necessary condition for the existence of a real-valued function $f$ on $A$ such that, for all $a, b \in A$, $a S b \Leftrightarrow f(a) \geq f(b)$. Together with the fact that $S$ is a weak order on $A$, it is also sufficient for the existence of such a representation (see Fishburn (1970) or Krantz et al. (1971)).

We say that $\succsim$ satisfies $O D_{i}^{*}$ if there is a finite or countably infinite set $A_{i} \subseteq X_{i}^{2}$ that is dense in $X_{i}^{2}$ for $\succsim_{i}^{*}$. Condition $O D^{*}$ is said to hold if condition $O D_{i}^{*}$ holds for all $i \in N$. This condition is trivially satisfied when $X_{i}$ is finite or countably infinite. In view of lemma 4 , it is necessary for $\left(\mathrm{M} 1^{\prime}\right)$. Together with the fact that all relations $\succsim_{i}^{*}$ are weak orders, it is also clearly sufficient for $\left(\mathrm{M}^{\prime}\right)$. The following example shows that $O D_{i}^{*}$ may not imply $O D_{j}^{*}$ for $j \neq i$.

\section{Example}

Let $X=X_{1} \times X_{2}$ with $X_{1}=\mathbb{R}^{2}$ and $X_{2}=\mathbb{R}$. Define $\succsim$ letting:

$$
\begin{aligned}
& x \succsim y \Leftrightarrow\left(\left(x_{1,1}, x_{1,2}\right), x_{2}\right) \succsim\left(\left(y_{1,1}, y_{1,2}\right), y_{2}\right) \Leftrightarrow \\
& \left\{\begin{array}{l}
\left(x_{1,1}-y_{1,1}\right)+\left(x_{2}-y_{2}\right)>0 \\
\text { or } \\
\left\{\begin{array}{l}
\left(x_{1,1}-y_{1,1}\right)+\left(x_{2}-y_{2}\right)=0 \\
\text { and } \\
\left(x_{1,2}-y_{1,2}\right)+\left(x_{2}-y_{2}\right) \geq 0 .
\end{array}\right.
\end{array}\right.
\end{aligned}
$$

It is easily shown that $\succsim_{1}^{*}$ is complete and such that: 


$$
\begin{array}{r}
\left(\left(x_{1,1}, x_{1,2}\right),\left(y_{1,1}, y_{1,2}\right)\right) \succsim_{1}^{*}\left(\left(z_{1,1}, z_{1,2}\right),\left(z_{1,1}, z_{1,2}\right)\right) \Leftrightarrow \\
\left\{\begin{array}{l}
x_{1,1}-y_{1,1}>z_{1,1}-w_{1,1} \\
\text { or } \\
\left\{\begin{array}{l}
x_{1,1}-y_{1,1}=z_{1,1}-w_{1,1} \\
\text { and } \\
x_{1,2}-y_{1,2} \geq z_{1,2}-w_{1,2},
\end{array}\right.
\end{array}\right.
\end{array}
$$

so that $O D_{1}^{*}$ is violated (see e.g. Fishburn (1970)) while we have $\left(x_{2}, y_{2}\right) \succsim_{2}^{*}$ $\left(z_{2}, w_{2}\right) \Leftrightarrow\left[x_{2}-y_{2} \geq z_{2}-w_{2}\right]$ so that $O D_{2}^{*}$ clearly holds.

We now turn to the case of models (M2'), (M3) and (M3'). Suppose that $\succsim_{i}^{* *}$ has a (skew symmetric) numerical representation so that there is a finite or countably infinite set $A_{i} \subseteq X_{i}^{2}$ that is dense in $X_{i}^{2}$ for $\succsim_{i}^{* *}$. Suppose that $\left(x_{i}, y_{i}\right) \succ_{i}^{*}\left(z_{i}, w_{i}\right)$. Since $\succsim_{i}^{* *}$ is complete, we have $\left(x_{i}, y_{i}\right) \succ_{i}^{* *}\left(z_{i}, w_{i}\right)$. This implies $\left(x_{i}, y_{i}\right) \succsim_{i}^{* *}\left(k_{i}, \ell_{i}\right)$ and $\left(k_{i}, \ell_{i}\right) \succsim_{i}^{* *}\left(z_{i}, w_{i}\right)$ for some $\left(k_{i}, \ell_{i}\right) \in A_{i}$. This in turn implies $\left(x_{i}, y_{i}\right) \succsim_{i}^{*}\left(k_{i}, \ell_{i}\right)$ and $\left(k_{i}, \ell_{i}\right) \succsim_{i}^{*}\left(z_{i}, w_{i}\right)$ so that $A_{i}$ is dense in $X_{i}^{2}$ for $\succsim_{i}^{*}$ and $O D_{i}^{*}$ holds. Hence, in view of lemma $4, O D^{*}$ is a necessary condition for models (M2'), (M3) and (M3'). It is not difficult to see that it is also sufficient when supplemented with the appropriate conditions used in theorem 1. Indeed, if the weak order $\succsim_{i}^{*}$ has a numerical representation $p_{i}$, the function $q_{i}$ defined letting $q_{i}\left(x_{i}, y_{i}\right)=p_{i}\left(x_{i}, y_{i}\right)-p_{i}\left(y_{i}, x_{i}\right)$ is a numerical representation of $\succsim_{i}^{* *}$.

We omit the cumbersome and apparently uninformative reformulation of $C^{*}$ and $O D^{*}$ in terms of $\succsim$. Theorem 1, lemma 4 and the preceding observations prove the central result in this paper:

Theorem 2 Let $\succsim$ be a binary relation on a set $X=\prod_{i=1}^{n} X_{i}$. Then:

1. $\succsim$ satisfies model $(M)$ iff it satisfies $C^{*}$,

2. ¿ satisfies model (M0) iff it is reflexive, independent and satisfies $C^{*}$,

3. ¿ satisfies model $\left(M 1^{\prime}\right)$ iff it is reflexive, independent and satisfies $R C 1$ and $O D^{*}$,

4. $\succsim$ satisfies model (M2') iff it is reflexive and satisfies $R C 1, R C 2$ and $O D^{*}$,

5. ¿ satisfies model (M3) iff it is complete and satisfies RC1, RC2 and $O D^{*}$ 


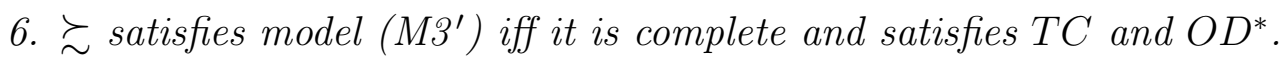

In view of lemmas 2 and 4 , we obtain the following:

Corollary 1 We have:

1. Models (M1) and (M1') are equivalent,

2. Models (M2) and (M2') are equivalent.

\subsection{Remarks}

The previous results prompt a number of remarks.

1. Parts 1, 2 and 3 of theorem 2 and the equivalence between (M1) and $\left(\mathrm{M1}^{\prime}\right)$ were already noted by Goldstein (1991), under a slightly different form. He also studies some variants of these models that are not dealt with here.

2. Lemma 2 combined with theorem 2 shows that all the models characterized are indeed different. We provide examples of each type of models in appendix.

3. It is not difficult to show that, when $\succsim$ is complete, $[R C 1, R C 2$ and $\left(x \sim y\right.$ and $\left.\left.\left(z_{i}, w_{i}\right) \succ_{i}^{* *}\left(x_{i}, y_{i}\right) \Rightarrow\left(z_{i}, x_{-i}\right) \succ\left(w_{i}, y_{-i}\right)\right)\right] \Leftrightarrow T C$. This offers an additional interpretation of $T C$ and shows that the only difference between (M3) and (M3') is the possible failure in (M3) of "strict monotonicity" with respect to $\succ_{i}^{* *}$ for pairs such that $x \sim y$ (see lemma 2). Furthermore, it is worth noting that (M3') is the only of our models for which there are close connections between partial preference relations and relations comparing preference differences on each attribute. Indeed, we have $x_{i} \succsim_{i} y_{i} \Leftrightarrow p_{i}\left(x_{i}, y_{i}\right) \geq 0$ so that $x_{i} \succsim_{i} y_{i} \Leftrightarrow\left(x_{i}, y_{i}\right) \succsim_{i}^{* *}\left(y_{i}, y_{i}\right)$. This explains why $\left(\mathrm{M} 3^{\prime}\right)$ was found in proposition 1 to be the only of our models for which there are nice monotonicity properties linking $\succsim$ and $\succsim_{i}$.

4. A word on the uniqueness of the numerical representations in theorem 2 is in order. As should be expected, the uniqueness of our representations is very weak; it is even weaker than the uniqueness of the representations of the transitive decomposable model (2) (Krantz et al., 
1971, Chapter 7). Indeed, it should be obvious from the proof of theorem 1 that there is much freedom in the choice of $F$ and $p_{i}$ when a representation exists. We take the example of model $\left(\mathrm{M} 1^{\prime}\right)$. It is clear that, in equation (6), the combination of:

- any representation $p_{i}$ of $\succsim_{i}^{*}$,

- any function $g$ from $\mathbb{R}^{n}$ to $\mathbb{R}$ increasing in all its arguments and

- any increasing function $f$ from $\mathbb{R}$ into $(0,+\infty)$,

leads to an acceptable representation. The situation is even worse remembering that in $\left(\mathrm{M} 1^{\prime}\right)$ it is not necessarily true that:

$$
p_{i}\left(x_{i}, y_{i}\right) \geq p_{i}\left(z_{i}, w_{i}\right) \Leftrightarrow\left(x_{i}, y_{i}\right) \succsim_{i}^{*}\left(z_{i}, w_{i}\right) .
$$

Therefore we will not try to explicitly formulate what would be the, obviously very awkward, conditions relating one representation to another in each of our models (thus, keeping in line with the idea that these numerical representations are not studied for their own sake and are only used in order to understand the pure consequences of our cancellation conditions).

Uniqueness results may however be obtained if the ordered set on which the numerical representations are sought is restricted from $\mathbb{R}$ to a much poorer subset. Consider the particular case of model (M1). Let $\left\langle F, p_{i}\right\rangle$ be a representation of $\succsim$ in (M1). Let $\phi$ be a nondecreasing function on $\mathbb{R}$ mapping $(-\infty, 0)$ to $\alpha<0$ and $[0,+\infty)$ to $\beta \geq 0$. It is clear that $\left\langle\phi \circ F, p_{i}\right\rangle$ is another representation in (M1). We henceforth restrict our attention to representations in (M1) such that the codomain of $F$ is $\{\alpha, \beta\}$ for some $\alpha<0$ and $\beta \geq 0$. We furthermore impose that these representations are regular, i.e. such that each $p_{i}$ is a numerical representation of $\succsim_{i}^{*}$. Given these additional restrictions, it is not difficult to devise a uniqueness result. Consider two representations $\left\langle F, p_{i}\right\rangle$ and $\left\langle G, q_{i}\right\rangle$ of $\succsim$ in (M1) satisfying our additional assumptions. It is clear that for all $i \in N$ there is an increasing function $\varphi_{i}$ on $\mathbb{R}$ such that $\varphi(0)=0$ for which $p_{i}=\varphi_{i}\left(q_{i}\right)$. Furthermore, $F$ can be deduced from $G$ letting $F\left(p_{1}, p_{2}, \ldots, p_{n}\right)=G\left(\varphi_{1}^{-1}\left(p_{1}\right), \varphi_{2}^{-1}\left(p_{2}\right) \ldots, \varphi_{n}^{-1}\left(p_{n}\right)\right)$.

A similar analysis can easily be conducted with (M2) and (M3): in (M2), it suffices to consider $\succsim_{i}^{* *}$ in lieu of $\succsim_{i}^{*}$ and increasing odd functions $\varphi_{i}$; in (M3), it suffices to consider representations in which the 
codomain of $F$ is $\{-\beta, 0, \beta\}$ for some $\beta \neq 0$. The situation is more complex with $\left(\mathrm{M} 3^{\prime}\right)$.

5. It should be observed that $R C 1$ and $O D^{*}$ are necessary and sufficient conditions to obtain a model in which:

$$
x \succsim y \Leftrightarrow F\left(p_{1}\left(x_{1}, y_{1}\right), p_{2}\left(x_{2}, y_{2}\right), \ldots, p_{n}\left(x_{n}, y_{n}\right)\right) \geq 0
$$

where $F$ is increasing in all its arguments. This is easily shown observing that, in the proof of part 3 of theorem 2 , independence of $\succsim$ is only used to obtain that $p_{i}\left(x_{i}, x_{i}\right)=0$, reflexivity implying that $F(\mathbf{0}) \geq 0$. Since model (9) encompasses relations $\succsim$ that are neither reflexive nor irreflexive, its interpretation is however subject to caution. This model seems more interesting when applied to an asymmetric binary relation interpreted as a strict preference relation. We do not explore this point here (see Bouyssou and Pirlot (2002)).

6. We already noticed that $R C 1_{i}$ amounts to defining a biorder between the sets $X_{i}^{2}$ and $X_{-i}^{2}$. Therefore $R C 1_{i}$ on its own implies, when $X$ is finite or countably infinite, the existence of two real-valued functions $p_{i}$ and $P_{-i}$ respectively on $X_{i}^{2}$ and $X_{-i}^{2}$ such that, for all $x, y \in X, x \succsim y$ iff $p_{i}\left(x_{i}, y_{i}\right)+P_{-i}\left(x_{-i}, y_{-i}\right) \geq 0$ (Ducamp \& Falmagne, 1969, Proposition $3)$. When supplemented with an appropriate density condition, $R C 1_{i}$ implies a similar result for sets of arbitrary cardinality (Doignon et al., 1984, Proposition 8). Therefore nontransitive additive conjoint models closely relate to ordinal measurement when $n=2$.

7. In a similar vein, Bouyssou (1986, Theorem 1) noted an interesting implication of $T C_{i}$ on its own. When $X$ is finite or countably infinite $T C_{i}$ implies the existence of two real-valued skew symmetric functions $p_{i}$ and $P_{-i}$ respectively on $X_{i}^{2}$ and $X_{-i}^{2}$ such that, for all $x, y \in X$, $x \succsim y \Leftrightarrow p_{i}\left(x_{i}, y_{i}\right)+P_{-i}\left(x_{-i}, y_{-i}\right) \geq 0$. As in remark 6 , this result can easily be extended to sets of arbitrary cardinality. When $n=2$ this offers an alternative to Fishburn (1991a, theorem B).

8. We already mentioned that the extension of $R C 1$ to subsets of attributes is the main necessary condition used by Vind (1991) together with topological assumptions on $X$ to axiomatize model (4) with $p_{i}\left(x_{i}\right.$, $\left.x_{i}\right)=0$. This prompts two remarks. First observe that imposing the 
generalization of $R C 1$ to all subsets of attributes does not imply independence. In Vind's result independence obtains from a complex synergy between the necessary conditions and his, unnecessary, structural assumptions (and, in particular, his condition A). Secondly, it may be interesting to observe the parallel between the axiomatization of (1) and (2) on the one side and (4) with $p_{i}\left(x_{i}, x_{i}\right)=0$ and (9) on the other. Additivity obtains in (1) when independence is combined with transitivity, completeness and structural assumptions. Decomposability in (2) results by keeping completeness and transitivity, dropping structural assumptions and restricting independence to weak independence, i.e. a condition similar to independence but only applied one attribute at a time. In the process of going from (1) to (2) the nice uniqueness result obtained with (1) was lost, whereas the proofs were much simpler. A surprisingly similar process is at work when going from (4) to (9). In the first case $R C 1$ is generalized to subsets of attributes together with structural assumptions; the resulting functions $p_{i}$ are unique up to the multiplication by a common positive constant. Dropping structural assumptions and using $R C 1$ results in a decomposable model without nice associated uniqueness result.

Similar remarks apply with skew symmetric and odd models when comparing part 6 of theorem 2 with Fishburn (1991b, Theorem C) or Fishburn (1990b, Theorem 1). The latter two results use a condition implying the generalization of $T C$ to subsets of attributes and impose structural assumptions on $X$. This leads to the skew symmetric version of (4) together with functions $p_{i}$ unique up to the multiplication by a common positive constant. We use $T C$ and drop all structural assumptions to obtain model (M3') for which there is no remarkable uniqueness property.

9. The various models studied in this paper allow us to draw the following picture of conjoint measurement models.

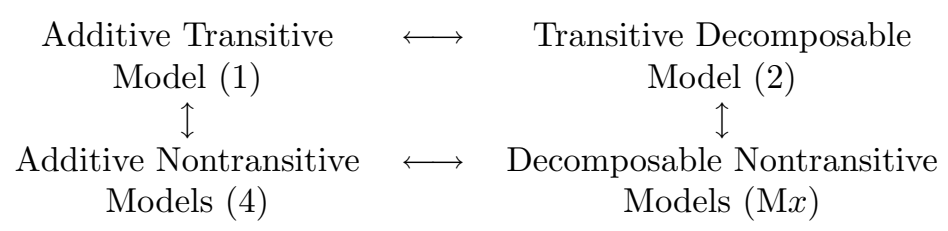

We analyze below how the various models in this diagram are related. 
- The connections between models (1) and (2) are elucidated in Krantz et al. (1971, Chapters 6 and 7). When $n \geq 3$, going from model (1) to model (2) amounts to replacing independence by weak independence and replacing the archimedean and solvability assumptions (or continuity and topological assumptions) by the requirement that the weak order $\succsim$ has a numerical representation.

- The connections between model (1) and the various models going under (4) (depending on the properties of the functions $p_{i}$ ) have been well studied in Fishburn (1990b, 1991b, 1992a) and Vind (1991). We take here the example of the skew symmetric version of (4) in which $\succsim$ is complete.

When $n=2$ model (4) is significantly different from model (1) since it relates more to ordinal than to conjoint measurement.

When $n \geq 3$, in the finite case, results for (1) and (4) are remarkably similar both using a denumerable set of necessary and sufficient conditions. The only, but essential, difference being that only cancellation conditions unrelated with transitivity are used in the characterization of model (4) (compare e.g. Fishburn (1970, Theorem 4.1.C) with Fishburn (1991b, Theorem A)).

In the general case with $n \geq 3$, the characterization of both models appeals to unnecessary structural assumptions. Although the structural assumptions needed for models of type (4) may be slightly different from the assumptions needed for model (1) (being generally stronger), Fishburn (1990b, 1991b) show that adding transitivity to the other conditions used in these results precipitates model (1). When proper structural assumptions are used, nice uniqueness results obtain in both models (with $p_{i}$ unique up to the multiplication by a common positive constant in (4) and $u_{i}$ defining interval scales with a common unit in (1)).

- When investigating the links between model (2) and the models of type (M), it should be observed that (2) does not imply any of $R C 1, R C 2$ and $T C$ (examples are easily built using a polynomial representation of the type $(x+y) \times z$ in $(2))$. It thus seems that the easiest way to connect both types of models is to start with the, almost, trivial model (M) and add to it completeness, transitivity and weak independence.

- As mentioned in remark 8 above, the connections between models 
of type (4) and models of type (M) are easily established. For instance going from $\left(\mathrm{M}^{\prime}\right)$ to the skew symmetric version of (4) mainly amounts to imposing a condition implying the generalization of $T C$ to subsets of attributes and adding adequate structural assumptions (compare Fishburn (1991b, Theorem C) with part 6 of theorem 2). For clear reasons, in the $n=2$ case both type of models are equivalent. The same is true comparing model (M1') with the version of (4) with $p_{i}\left(x_{i}, y_{i}\right)=0$ studied in Vind (1991), although in this last result the interactions between necessary conditions (the generalization of $R C 1$ to subsets of attributes) and the structural assumptions stated in topological terms seem very strong (implying independence).

10. When $n \geq 3$ most results on model (1) appeal to independence rather than TC (Debreu, 1960; Krantz et al., 1971). Although it is true that independence is a simpler condition that may be easier to test than $T C$, our results suggest to reconsider the role of independence as the "central condition" in conjoint measurement models. Indeed, theorem 2 shows that some cancellation conditions has much more "power" on their own (i.e. when analyzed without supposing any particular structure on $X$ and any other property for $\succsim$ ) than others (note that this is related to the comments of Furkhen and Richter (1991) concerning the difficulty to separate, in classical theorems analyzing (1), the respective roles of necessary structural conditions and the unnecessary structural assumptions). Although $T C$, in presence of reflexivity, is stronger than independence, its use leads:

- to avoid the asymmetry between the $n=2$ and the $n \geq 3$ cases in the analysis of model (1) (Wakker, 1989, Th.III.6.6.(iii)) and

- together with completeness, to the already rather well-structured model (M3') on sets having no particular structure.

11. A different line of specialization of $(M)$ and its extensions involves "intra-attribute decomposability", i.e. the specification of a particular functional form for the functions $p_{i}$. Let us notice that model (M) may equivalently, for finite or countably infinite $X$, be written as:

$$
x \succsim y \Leftrightarrow F\left(\phi_{1}\left(u_{1}\left(x_{1}\right), v_{1}\left(y_{1}\right)\right), \ldots, \phi_{n}\left(u_{n}\left(x_{n}\right), v_{n}\left(y_{n}\right)\right)\right) \geq 0
$$


where $u_{i}$ and $v_{i}$ are real-valued functions on $X_{i}$ and $\phi_{i}$ is a real-valued function on $u_{i}\left(X_{i}\right) \times v_{i}\left(X_{i}\right)$. To show how this is possible, define the binary relations $E_{i}^{R}$ and $E_{i}^{L}$ on $X_{i}$ letting for all $x_{i}, y_{i} \in X_{i}$ :

$$
\begin{aligned}
x_{i} E_{i}^{R} y_{i} & \Leftrightarrow\left(x_{i}, z_{i}\right) \sim_{i}^{*}\left(y_{i}, z_{i}\right), \text { for all } z_{i} \in X_{i}, \\
x_{i} E_{i}^{L} y_{i} & \Leftrightarrow\left(z_{i}, y_{i}\right) \sim_{i}^{*}\left(z_{i}, x_{i}\right), \text { for all } z_{i} \in X_{i} .
\end{aligned}
$$

It is clear that $E_{i}^{R}$ and $E_{i}^{L}$ are equivalence relations. Since $X$ has been supposed to be denumerable, there are real-valued functions $u_{i}$ and $v_{i}$ on $X_{i}$ so that, for all $x_{i}, y_{i} \in X_{i}$ :

$$
\begin{aligned}
& {\left[x_{i} E_{i}^{R} y_{i} \Leftrightarrow u_{i}\left(x_{i}\right)=u_{i}\left(y_{i}\right)\right] \text { and }} \\
& {\left[x_{i} E_{i}^{L} y_{i} \Leftrightarrow v_{i}\left(x_{i}\right)=v_{i}\left(y_{i}\right)\right] .}
\end{aligned}
$$

Given a particular representation of $\succsim$ in model (M), define $\phi_{i}$ on $u_{i}\left(X_{i}\right) \times v_{i}\left(X_{i}\right)$ letting, for all $x_{i}, y_{i} \in X_{i}, \phi_{i}\left(u\left(x_{i}\right), v\left(y_{i}\right)\right)=p_{i}\left(x_{i}, y_{i}\right)$. The well-definedness of $\phi_{i}$ easily follows from the definitions of $\sim_{i}^{*}, E_{i}^{R}$ and $E_{i}^{L}$.

Imposing additional properties on the functions $\phi_{i}$ (e.g. requiring that $u_{i} \equiv v_{i}$ or that $\phi_{i}$ is nondecreasing in its first argument and nonincreasing in its second argument) leads to nontrivial models that are studied in Bouyssou and Pirlot (2001). These additional conditions may be combined with the variety of models studied in this paper. This large variety of models will bring us closer to the additive difference model (3) while not invoking the full force "inter-attribute additivity" and "intra-attribute subtractivity". This gives rise to models that are "in between" (2) and (M) much like the additive difference model (3) is "in between" models (1) and (4). The intuition behind these intradecomposable models is that the "weight of the difference" between elements of $X_{i}$ (i.e. $\left.p_{i}\left(x_{i}, y_{i}\right)\right)$ may be understood via linear arrangements on these elements (through the functions $u_{i}$ and $v_{i}$ ).

\section{Discussion}

We hope, in the preceding section, to have convinced the reader that there may be a formal interest in studying "unconventional" representations of nontransitive relations. Apart from this formal interest, let us mention that: 
- The various cancellation conditions used in theorem 2 appear to be easily subjected to empirical tests. In view of our results we are inclined to consider that $R C 1, R C 2$ and $T C$ qualify as central conditions for conjoint measurement models whether or not they are transitive or complete. This calls for empirical future research.

- The various models studied in this paper were shown in Greco, Matarazzo, and Słowiński (1999a, 1999b) to have close connections with preference models representable by "IF ... THEN ..." rules that frequently arise in Artificial Intelligence.

- As already discussed in Goldstein (1991), our models are flexible enough to encompass many aggregation rules that have been proposed in the literature, e.g. additive utility, additive differences, (weighted) majority or greatest attractiveness difference (Russo \& Dosher, 1983; Huber, 1979; Dahlstrand \& Montgomery, 1984; Montgomery \& Svenson, 1976; Svenson, 1979; Payne, Bettman, \& Johnson, 1988; Ball, 1997; Aschenbrenner, 1981; Aschenbrenner, Albert, \& Schalhofer, 1984).

- Our framework is sufficiently general to encompass "compensatory" as well as "noncompensatory" preference relations, e.g. a preference based on a weighted sum and a preference based on a lexicographic rule. As shown in Bouyssou and Pirlot (2002), this leads to a characterization of noncompensatory preferences avoiding the use of highly specific conditions as done in Fishburn (1976) and Bouyssou and Vansnick (1986) and gives clues on how to define the "degree of compensatoriness" of a preference relation.

Future research on the topics discussed in this paper will include:

- the study of various "intra-decomposable" versions of our models (Bouyssou \& Pirlot, 2001) exploring particular functional forms for the $p_{i}$,

- the generalization of our results to aggregation methods leading to valued preference relations (Bouyssou \& Pirlot, 1999; Bouyssou, Pirlot, \& Vincke, 1997; Pirlot \& Vincke, 1997),

- the specialization of our results to the case in which $X$ is an homogeneous Cartesian product $\left(X_{i}=X_{j}, \forall i, j \in N\right)$ which includes the important case of decision under uncertainty (Bouyssou, Perny, \& Pirlot, 2000), 
- the study of additional conditions making possible to specify a precise functional form for $F$ (e.g. min or max).

\section{Appendix: Examples}

We provide below simple examples showing that models $(\mathrm{M} 0),\left(\mathrm{M}^{\prime}\right),\left(\mathrm{M} 2^{\prime}\right)$, (M3) and (M3') are indeed different.

\section{Example 1}

Let $X=\mathbb{R}^{2}$ and, for all $x, y \in X, x \succsim y \Leftrightarrow F\left(p_{1}\left(x_{1}, y_{1}\right), p_{2}\left(x_{2}, y_{2}\right)\right) \geq 0$, with $p_{i}\left(x_{i}, y_{i}\right)=x_{i}-y_{i}$ and $F\left(p_{1}, p_{2}\right)=p_{1} \times p_{2}$.

By construction, $\succsim$ has a representation in model (M0). It is easy to see that $\succsim$ violates $R C 1$ and, thus, cannot be represented in (M1').

\section{Example 2}

Let $X=\mathbb{R}^{2}$ and, for all $x, y \in X, x \succsim y \Leftrightarrow F\left(p_{1}\left(x_{1}, y_{1}\right), p_{2}\left(x_{2}, y_{2}\right)\right) \geq 0$, with $p_{2}\left(x_{2}, y_{2}\right)=x_{2}-y_{2}, p_{1}\left(x_{1}, y_{1}\right)=\left\{\begin{array}{l}x_{1}-y_{1} \text { if } x_{1} \geq y_{1}, \\ \left|x_{1}\right|\left(x_{1}-y_{1}\right) \text { otherwise, }\end{array}\right.$ and $F\left(p_{1}, p_{2}\right)=p_{1}+p_{2}$.

By construction, $\succsim$ has a representation in model $\left(\mathrm{M} 1^{\prime}\right)$.

Observe that $\succsim_{1}^{* *}$ is not complete since neither $(3 ; 1) \succsim_{1}^{* *}(11 ; 10)$ nor $(11 ; 10) \succsim_{1}^{* *}(3 ; 1)$. Indeed there are $\left(x_{2}, y_{2}\right)$ such that $\left[\left(1, x_{2}\right) \succsim\left(3, y_{2}\right)\right]$ and $\operatorname{Not}\left[\left(10, x_{2}\right) \succsim\left(11, y_{2}\right)\right]$, which implies $\operatorname{Not}\left[(3 ; 1) \succsim_{1}^{*}(11 ; 10)\right]$ so that $\operatorname{Not}\left[(3 ; 1) \succsim_{1}^{* *}(11 ; 10)\right]$. Similarly, there are $\left(x_{2}, y_{2}\right)$ such that $\left[\left(3, x_{2}\right) \succsim\right.$ $\left.\left(1, y_{2}\right)\right]$ and $\operatorname{Not}\left[\left(11, x_{2}\right) \succsim\left(10, y_{2}\right)\right]$, which implies $\operatorname{Not}\left[(11 ; 10) \succsim_{1}^{*}(3 ; 1)\right]$ so that $\operatorname{Not}\left[(11 ; 10) \succsim_{1}^{* *}(3 ; 1)\right]$. Hence $\succsim$ violates $R C 2$ and cannot be represented in $\left(\mathrm{M} 2^{\prime}\right)$.

\section{Example 3}

Let $X=\mathbb{R}^{2}$ and, for all $x, y \in X, x \succsim y \Leftrightarrow F\left(p_{1}\left(x_{1}, y_{1}\right), p_{2}\left(x_{2}, y_{2}\right)\right) \geq 0$, with $p_{i}\left(x_{i}, y_{i}\right)=\frac{2}{\Pi} \arctan \left(x_{i}-y_{i}\right)$ and $F\left(p_{1}, p_{2}\right)=p_{1}+p_{2}+p_{1} p_{2}$.

It is easily checked that $\succsim$ satisfies model (M2') since all functions $p_{i}$ are skew symmetric and $F$ is increasing in all its arguments. The relation $\succsim$ is not complete (taking $(x, y)$ such that $p_{1}\left(x_{1}, y_{1}\right)=1 / 4$ and $p_{2}\left(x_{2}, y_{2}\right)=-1 / 4$, we have neither $\left(x_{1}, x_{2}\right) \succsim\left(y_{1}, y_{2}\right)$ nor $\left.\left(y_{1}, y_{2}\right) \succsim\left(x_{1}, x_{2}\right)\right)$. Hence $\succsim$ cannot be represented in model (M3). 


\section{Example 4}

Let $X=\mathbb{R}^{2}$ and, for all $x, y \in X, x \succsim y \Leftrightarrow F\left(p_{1}\left(x_{1}, y_{1}\right), p_{2}\left(x_{2}, y_{2}\right)\right) \geq 0$, with $p_{i}\left(x_{i}, y_{i}\right)=x_{i}-y_{i}$ and $F\left(p_{1}, p_{2}\right)=\left\{\begin{array}{l}p_{1}+p_{2} \text { if }\left|p_{1}+p_{2}\right| \geq 1 \\ 0 \text { otherwise. }\end{array}\right.$

By construction, $\succsim$ has a representation in (M3). Simple examples show that $\succsim$ violates $T C$ so that it cannot be represented in $\left(\mathrm{M} 3^{\prime}\right)$.

\section{References}

Aschenbrenner, K.M. (1981). Efficient sets, decision heuristics and singlepeaked preferences. Journal of Mathematical Psychology, 23, 227-256.

Aschenbrenner, K.M., Albert, D., \& Schalhofer, F. (1984). Stochastic choice heuristics. Acta Psychologica, 56, 153-166.

Ball, C. (1997). A comparison of single-step and multiple-step transition analyses of multiattribute decision strategies. Organisational Behavior and Human Decision Processes, 69, 195-204.

Bouyssou, D. (1986). Some remarks on the notion of compensation in MCDM. European Journal of Operational Research, 26, 150-160.

Bouyssou, D., Perny, P., \& Pirlot, M. (2000). Nontransitive decomposable conjoint measurement as a general framework for $M C D M$ and decision under uncertainty. (Communication to EURO XVII, Budapest, Hungary, 16-19 July)

Bouyssou, D., \& Pirlot, M. (1999). Conjoint measurement without additivity and transitivity. In N. Meskens \& M. Roubens (Eds.), Advances in decision analysis (pp. 13-29). Dordrecht: Kluwer.

Bouyssou, D., \& Pirlot, M. (2001). 'Additive difference' models without additivity and subtractivity. (Working Paper)

Bouyssou, D., \& Pirlot, M. (2002). A characterization of strict concordance relations. In D. Bouyssou, E. Jacquet-Lagrèze, P. Perny, R. Słowiński, D. Vanderpooten, \& Ph. Vincke (Eds.), Aiding decisions with multiple criteria: Essays in honour of Bernard Roy (pp. 121-145). Dordrecht: Kluwer. 
Bouyssou, D., Pirlot, M., \& Vincke, Ph. (1997). A general model of preference aggregation. In M.H. Karwan, J. Spronk, \& J. Wallenius (Eds.), Essays in decision making (pp. 120-134). Berlin: Springer Verlag.

Bouyssou, D., \& Vansnick, J.-C. (1986). Noncompensatory and generalized noncompensatory preference structures. Theory and Decision, 21, 251266.

Chipman, J.S. (1971). Consumption theory without transitive indifference. In J.S. Chipman, L. Hurwicz, M.K. Richter, \& H. Sonnenschein (Eds.), Studies in the mathematical foundations of utility and demand theory: A symposium at the University of Michigan, 1968 (pp. 224-253). Harcourt-Brace.

Croon, M.A. (1984). The axiomatization of additive difference models for preference judgements. In E. Degreef \& G. van Buggenhaut (Eds.), Trends in mathematical psychology (pp. 193-227). Amsterdam: NorthHolland.

Dahlstrand, V., \& Montgomery, H. (1984). Information search and evaluation processes in decision-making: A computer-based process tracking study. Acta Psychologica, 56, 113-123.

Debreu, G. (1960). Topological methods in cardinal utility theory. In S. K. K.J. Arrow \& P. Suppes (Eds.), Mathematical methods in the social sciences (pp. 16-26). Stanford: Stanford University Press.

Doignon, J.-P., Ducamp, A., \& Falmagne, J.-C. (1984). On realizable biorders and the biorder dimension of a relation. Journal of Mathematical Psychology, 28, 73-109.

Ducamp, A., \& Falmagne, J.-C. (1969). Composite measurement. Journal of Mathematical Psychology, 6, 359-390.

Fishburn, P.C. (1970). Utility theory for decision-making. New-York: Wiley.

Fishburn, P.C. (1976). Noncompensatory preferences. Synthese, 33, 393-403.

Fishburn, P.C. (1980). Lexicographic additive differences. Journal of Mathematical Psychology, 21, 191-218. 
Fishburn, P.C. (1982). Nontransitive measurable utility. Journal of Mathematical Psychology, 26, 31-67.

Fishburn, P.C. (1985). Interval orders and intervals graphs. New-York: Wiley.

Fishburn, P.C. (1988). Nonlinear preference and utility theory. Baltimore: Johns Hopkins University Press.

Fishburn, P.C. (1990a). Additive non-transitive preferences. Economic Letters, 34, 317-321.

Fishburn, P.C. (1990b). Continuous nontransitive additive conjoint measurement. Mathematical Social Sciences, 20, 165-193.

Fishburn, P.C. (1990c). Skew symmetric additive utility with finite states. Mathematical Social Sciences, 19, 103-115.

Fishburn, P.C. (1991a). Nontransitive preferences in decision theory. Journal of Risk and Uncertainty, 4, 113-134.

Fishburn, P.C. (1991b). Nontransitive additive conjoint measurement. Journal of Mathematical Psychology, 35, 1-40.

Fishburn, P.C. (1992a). Additive differences and simple preference comparisons. Journal of Mathematical Psychology, 36, 21-31.

Fishburn, P.C. (1992b). On nonstandard nontransitive additive utility. Journal of Economic Theory, 56, 426-433.

Fishburn, P.C. (1996). Finite linear qualitative probability. Journal of Mathematical Psychology, 40, 21-31.

Fishburn, P.C. (1997). Cancellation conditions for multiattribute preferences on finite sets. In M.H. Karwan, J. Spronk, \& J. Wallenius (Eds.), Essays in decision making (pp. 157-167). Berlin: Springer Verlag.

Furkhen, G., \& Richter, M.K. (1991). Additive utility. Economic Theory, 1, 83-105.

Gilboa, I., \& Lapson, R. (1995). Aggregation of semiorders: Intransitive indifference makes a difference. Economic Theory, 5, 109-126. 
Goldstein, W.M. (1991). Decomposable threshold models. Journal of Mathematical Psychology, 35, 64-79.

Gonzales, Ch. (1996). Additive utilities when some components are solvable and others not. Journal of Mathematical Psychology, 40, 141-151.

Gonzales, Ch. (2000). Two factor additive conjoint measurement with one solvable component. Journal of Mathematical Psychology, 44, 285-309.

Greco, S., Matarazzo, B., \& Słowiński, R. (1999a). Rough approximation of a preference relation by dominance relations. European Journal of Operational Research, 117, 63-83.

Greco, S., Matarazzo, B., \& Słowiński, R. (1999b). The use of rough sets and fuzzy sets in MCDM. In T. Gal, T. Hanne, \& T. Stewart (Eds.), Multicriteria decision making, Advances in MCDM models, algorithms, theory and applications (pp. 14.1-14.59). Kluwer.

Huber, O. (1979). Non transitive multidimensional preferences: Theoretical analysis of a model. Theory and Decision, 10, 147-165.

Iverson, G., \& Falmagne, J.-C. (1985). Statistical issues in measurement. Mathematical Social Sciences, 10, 131-153.

Jaffray, J.-Y. (1974). On the extension of additive utilities to infinite sets. Journal of Mathematical Psychology, 11, 431-452.

Karni, E., \& Safra, Z. (1998). The hexagon condition and additive representation for two dimensions: An algebraic approach. Journal of Mathematical Psychology, 42, 393-399.

Kim, T., \& Richter, M.K. (1986). Nontransitive-nontotal consumer theory. Journal of Economic Theory, 38, 324-363.

Krantz, D.H., Luce, R.D., Suppes, P., \& Tversky, A. (1971). Foundations of measurement, vol. 1: Additive and polynomial representations. NewYork: Academic Press.

Luce, R.D. (1978). Lexicographic tradeoff structures. Theory and Decision, 9, 187-193. 
Luce, R.D., Krantz, D.H., Suppes, P., \& Tversky, A. (1990). Foundations of measurement, vol. 3: Representation, axiomatisation and invariance. New-York: Academic Press.

May, K.O. (1954). Intransitivity, utility and the aggregation of preference patterns. Econometrica, 22, 1-13.

Montgomery, H., \& Svenson, O. (1976). On decision rules and information processing strategies for choice among multiattribute alternatives. Scandinavian Journal of Psychology, 17, 283-291.

Nakamura, Y. (1997). Lexicographic additivity for multi-attribute preferences on finite sets. Theory and Decision, 42, 1-19.

Payne, J.W., Bettman, J.R., \& Johnson, E.J. (1988). Adaptative strategy selection in decision making. Journal of Experimental Psychology: Learning, Memory and Cognition, 14, 534-552.

Pirlot, M., \& Vincke, Ph. (1997). Semiorders. Properties, representations, applications. Dordrecht: Kluwer.

Roberts, F.S. (1979). Measurement theory with applications to decision making, utility and the social sciences. Reading: Addison-Wesley.

Russo, J.E., \& Dosher, B.A. (1983). Strategies for multiattribute binary choice. Journal of Experimental Psychology: Learning, Memory and Cognition, 9, 676-696.

Scott, D. (1964). Measurement structures and linear inequalities. Journal of Mathematical Psychology, 1, 233-247.

Suppes, P., Krantz, D.H., Luce, R.D., \& Tversky, A. (1989). Foundations of measurement, vol. 2: Geometrical, threshold, and probabilistic representations. Academic Press, New York.

Svenson, O. (1979). Process description of decision making. Organizational Behavior and Human Performance, 23, 86-112.

Tversky, A. (1969). Intransitivity of preferences. Psychological Review, 76, $31-48$. 
Vind, K. (1991). Independent preferences. Journal of Mathematical Economics, 20, 119-135.

Wakker, P.P. (1988). Derived strength of preference relations on coordinates. Economic Letters, 28, 301-306.

Wakker, P.P. (1989). Additive representations of preferences: A new foundation of decision analysis. Dordrecht: Kluwer. 